\title{
MINIMAL THINNESS WITH RESPECT TO SYMMETRIC LÉVY PROCESSES
}

\author{
PANKI KIM, RENMING SONG, AND ZORAN VONDRAČEK
}

\begin{abstract}
Minimal thinness is a notion that describes the smallness of a set at a boundary point. In this paper, we provide tests for minimal thinness at finite and infinite minimal Martin boundary points for a large class of purely discontinuous symmetric Lévy processes.
\end{abstract}

\section{INTRODUCTION}

Minimal thinness is a notion that describes the smallness of a set at a boundary point. Minimal thinness in the half-space was introduced by Lelong-Ferrand in 35, while minimal thinness in general open sets was developed by Naïm in [38; for a more recent exposition see [4, Chapter 9]. A probabilistic interpretation in terms of Brownian motion was given by Doob; see e.g. [19].

A Wiener-type criterion for minimal thinness of a subset of the half-space (using a Green energy) has already appeared in [35. A refined version of such a criterion (using the ordinary capacity) was proved in [20]. A general version of the Wienertype criterion for minimal thinness in NTA domains was established by Aikawa in [1] using a powerful concept of quasi-additivity of capacity. In case of a smooth domain, Aikawa's version of the criterion implies several results obtained earlier; cf. [5, 18, 36, 41]. A good exposition of this theory can be found in [2, Part II, 7].

All of these results have been proved in the context of classical potential theory related to the Laplacian or, probabilistically, to Brownian motion. Even though the concept of minimal thinness for Hunt processes admitting a dual process (and satisfying an additional hypothesis) was studied by Föllmer [22, concrete criteria for minimal thinness with respect to certain integro-differential operators (i.e., certain Lévy processes) in the half-space have been obtained only recently in 28 . To be more precise, in 28 the underlying process $X$ belongs to a class of subordinate Brownian motions, where the Laplace exponents of the corresponding subordinators are complete Bernstein functions satisfying a certain condition at infinity. The first

Received by the editors May 1, 2014 and, in revised form, October 5, 2014, November 1, 2014, and November 17, 2014.

2010 Mathematics Subject Classification. Primary 60J50, 31C40; Secondary 31C35, 60J45, $60 \mathrm{~J} 75$.

Key words and phrases. Minimal thinness, symmetric Lévy process, unimodal Lévy process, boundary Harnack principle, Green function, Martin kernel, quasi-additivity, Wiener-type criterion.

The work of Panki Kim was supported by the National Research Foundation of Korea (NRF) grant funded by the Korean government (MEST) (NRF-2013R1A2A2A01004822).

The research of Renming Song was supported in part by a grant from the Simons Foundation (208236).

The research of Zoran Vondraček was supported in part by the Croatian Science Foundation under the project 3526. 
result of [28] was a necessary condition for minimal thinness of a Borel subset $E$ of the half-space $\mathbb{H} \subset \mathbb{R}^{d}, d \geq 2$ : If $E$ is minimally thin in $\mathbb{H}$ (with respect to the process $X$ ) at the point $z=0$, then

$$
\int_{E \cap B(0,1)}|x|^{-d} d x<\infty .
$$

Here, and in the sequel, $B(z, r)$ denotes the open ball centered at $z \in \mathbb{R}^{d}$ with radius $r>0$. In the classical case this was proved in [5] for $d=2$ and in [18] for $d \geq 3$. The method applied in [28] was based on a result of Sjögren, [40, Theorem 2]. The second result of [28] was a criterion for minimal thinness in $\mathbb{H}$ of a set under the graph of a Lipschitz function, which in the classical case is due to Burdzy; see [11] and 25].

The goal of this paper is to generalize the results from 28] in several directions. We will always assume that $d \geq 2$. We work with a broader class of purely discontinuous symmetric transient Lévy processes and prove a version of Aikawa's Wiener-type criterion for minimal thinness of a subset of a (not necessarily bounded) $\kappa$-fat open set at any finite (minimal Martin) boundary point. By specializing to $C^{1,1}$ open sets, we get an integral criterion for minimal thinness in the spirit of [5, 18. Moreover, in case the processes satisfy an additional assumption governing the global behavior, we obtain criteria for minimal thinness of a subset of half-space-like open sets at infinity. In the classical case of the Laplacian, such results are direct consequences of the corresponding finite boundary point results by use of the inversion with respect to a sphere and the Kelvin transform. In the case we study, this is much more delicate, since the method of Kelvin transform is not available.

Let us describe the results of the paper in more detail. We start with a description of the setup of this paper.

We assume that $r \mapsto j(r)$ is a strictly positive non-increasing function on $(0, \infty)$ satisfying

$$
j(r) \leq c j(r+1) \quad \text { for } r \geq 1,
$$

and $X$ is a purely discontinuous symmetric transient Lévy process in $\mathbb{R}^{d}$ with Lévy exponent $\Psi_{X}(\xi)$ so that

$$
\mathbb{E}_{x}\left[e^{i \xi \cdot\left(X_{t}-X_{0}\right)}\right]=e^{-t \Psi_{X}(\xi)}, \quad t>0, x \in \mathbb{R}^{d}, \xi \in \mathbb{R}^{d} .
$$

We assume that the Lévy measure of $X$ has a density $J_{X}$ such that

$$
\gamma^{-1} j(|y|) \leq J_{X}(y) \leq \gamma j(|y|), \quad \text { for all } y \in \mathbb{R}^{d},
$$

for some $\gamma \geq 1$. Since $\int_{0}^{\infty} j(r)\left(1 \wedge r^{2}\right) r^{d-1} d r<\infty$ by (1.2), the function $x \rightarrow j(|x|)$ is the Lévy density of an isotropic unimodal Lévy process whose characteristic exponent is $\Psi(|\xi|)=\int_{\mathbb{R}^{d}}(1-\cos (\xi \cdot y)) j(|y|) d y$. The Lévy exponent $\Psi_{X}$ can be written as $\Psi_{X}(\xi)=\int_{\mathbb{R}^{d}}(1-\cos (\xi \cdot y)) J_{X}(y) d y$ and, clearly by (1.2), it satisfies

$$
\gamma^{-1} \Psi(|\xi|) \leq \Psi_{X}(\xi) \leq \gamma \Psi(|\xi|), \quad \text { for all } \xi \in \mathbb{R}^{d} .
$$

The function $\Psi$ may not be increasing. However, if we put $\Psi^{*}(r):=\sup _{s \leq r} \Psi(s)$, then, by [8, Proposition 2], we have

$$
\Psi(r) \leq \Psi^{*}(r) \leq \pi^{2} \Psi(r) .
$$


Thus by (1.3),

$$
\left(\pi^{2} \gamma\right)^{-1} \Psi^{*}(|\xi|) \leq \Psi_{X}(\xi) \leq \gamma \Psi^{*}(|\xi|), \quad \text { for all } \xi \in \mathbb{R}^{d} .
$$

Moreover,

$$
\Psi^{*}(\lambda t) \leq 2\left(1+\lambda^{2}\right) \Psi^{*}(t) \quad \text { for every } t>0 \text { and } \lambda \geq 1
$$

(see [26, Lemma 1]).

We will always assume that $\Psi$ satisfies the following scaling condition at infinity:

(H1): There exist constants $0<\delta_{1} \leq \delta_{2}<1$ and $a_{1}, a_{2}>0$ such that

$$
a_{1} \lambda^{2 \delta_{1}} \Psi(t) \leq \Psi(\lambda t) \leq a_{2} \lambda^{2 \delta_{2}} \Psi(t), \quad \lambda \geq 1, t \geq 1 .
$$

Then by [8, (15) and Corollary 22], for every $R>0$, there exists $c=c(R)>1$ such that

$$
c^{-1} \frac{\Psi\left(r^{-1}\right)}{r^{d}} \leq j(r) \leq c \frac{\Psi\left(r^{-1}\right)}{r^{d}} \text { for } r \in(0, R] .
$$

Note that the class of Lévy processes described above contains the purely discontinuous unimodal Lévy processes dealt with in [8,10]. The condition (H1) governs the small time and small space behavior of the process $X$. Thus it is sometimes referred to as a local condition.

In this paper, we will always assume that the condition $(\mathbf{H 1})$ is satisfied and $X$ is a purely discontinuous symmetric transient Lévy process with Lévy density $J_{X}$ satisfying (1.2).

To study minimal thinness at infinity, we need to add another scaling condition on $\Psi$ near the origin:

(H2): There exist constants $0<\delta_{3} \leq \delta_{4}<1$ and $a_{3}, a_{4}>0$ such that

$$
a_{3} \lambda^{2 \delta_{4}} \Psi(t) \leq \Psi(\lambda t) \leq a_{4} \lambda^{2 \delta_{3}} \Psi(t), \quad \lambda \leq 1, t \leq 1 .
$$

Since $d \geq 2,(\mathbf{H 2})$ implies that $X$ is transient. Condition (H2) governs the large time and large space behavior of $X$ and so it is sometimes referred to as a global condition.

We will impose the condition (H2) only when we discuss minimal thinness at infinity and we will explicitly mention this assumption when stating the results at the beginning of the section.

Let $(\mathcal{E}, \mathcal{F})$ be the Dirichlet form of $X$ on $L^{2}\left(\mathbb{R}^{d}, d x\right)$. It is known that $(\mathcal{E}, \mathcal{F})$ is a regular Dirichlet form on $L^{2}\left(\mathbb{R}^{d}, d x\right)$ and $\mathcal{F}=\overline{C_{c}^{\infty}\left(\mathbb{R}^{d}\right)} \mathcal{E}_{1}=\left\{f \in L^{2}\left(\mathbb{R}^{d}, d x\right)\right.$ : $\mathcal{E}(f, f)<\infty\}$, where $\mathcal{E}_{1}(u, u)=\mathcal{E}(u, u)+\int_{\mathbb{R}^{d}} u^{2}(x) d x$. Moreover, for $u \in \mathcal{F}$,

$$
\mathcal{E}(u, u)=\int_{\mathbb{R}^{d} \times \mathbb{R}^{d}}(u(x)-u(y))^{2} J_{X}(x-y) d x d y .
$$

For any open $D \subset \mathbb{R}^{d}$, we use $X^{D}=\left(X_{t}^{D}, \mathbb{P}_{x}\right)$ to denote the subprocess of $X$ killed upon exiting $D$. The Dirichlet form of $X^{D}$ is given by $\left(\mathcal{E}, \mathcal{F}_{D}\right)$, where

$$
\mathcal{F}_{D}=\left\{u \in \mathcal{F}: u=0 \text { on } D^{c} \text { except for a set of zero capacity }\right\} .
$$

The Hardy inequality is one of the main ingredients in Aikawa's construction of a measure comparable to the capacity, which is fundamental in proving quasiadditivity of capacity (see [1,2]). We introduce a local Hardy inequality for the Dirichlet form $\left(\mathcal{E}, \mathcal{F}_{D}\right)$ in the next definition and show in Section 5 that it holds under natural conditions on the open set $D$. 
Definition 1.1. We say that $\left(\mathcal{E}, \mathcal{F}_{D}\right)$ satisfies the local Hardy inequality at $z \in \partial D$ (with a localization constant $r_{0}$ ) if there exist $c>0$ and $r_{0}>0$ such that

$$
\mathcal{E}(v, v) \geq c \int_{B\left(z, r_{0}\right) \cap D} v^{2}(x) \Psi\left(\delta_{D}(x)^{-1}\right) d x, \quad v \in \mathcal{F}_{D} .
$$

We recall now the definition of $\kappa$-fat open set and introduce the necessary notation.

Definition 1.2. Let $0<\kappa \leq 1$. We say that an open set $D$ is $\kappa$-fat at $z \in \partial D$ if there is a localization radius $R>0$ such that for all $r \in(0, R]$ there exists a (nontangential) point $A_{r}(z) \in D \cap B(z, r)$ such that the ball $B\left(A_{r}(z), \kappa r\right) \subset D \cap B(z, r)$. We say that an open set is $\kappa$-fat with localization radius $R$ if $D$ is $\kappa$-fat at all $z \in \partial D$ with localization radius $R$.

Without loss of generality, we will assume that $R \leq 1 / 2$ and $\kappa \leq 1 / 4$.

The first main result of this paper is the following Aikawa's version of the Wienertype criterion for minimal thinness. For any open set $D \subset \mathbb{R}^{d}$, we use $G_{D}$ to denote the Green function of $X^{D}$. See Definition 6.1 for the definition of minimal thinness in $D$ with respect to $X$.

Theorem 1.3. Assume that $D \subset \mathbb{R}^{d}$ is $\kappa$-fat with localization radius $R$ and that $\left(\mathcal{E}, \mathcal{F}_{D}\right)$ satisfies the local Hardy inequality with a localization constant $r_{0}$ at $z \in \partial D$. Fix a point $x_{0} \in D$ with $\kappa R<\delta_{D}\left(x_{0}\right)<R$.

(1) If a Borel set $E \subset D$ is minimally thin in $D$ at $z$ with respect to $X$, then

$$
\int_{E \cap B\left(z,(\kappa R / 4) \wedge r_{0}\right)}\left(\frac{G_{D}\left(x, x_{0}\right)}{G_{D}\left(A_{|x-z|}(z), x_{0}\right)}\right)^{2} \frac{\Psi\left(\delta_{D}(x)^{-1}\right)}{\Psi\left(|x-z|^{-1}\right)} \frac{d x}{|x-z|^{d}}<\infty .
$$

(2) Conversely, if $E$ is the union of a subfamily of Whitney cubes of $D$ and is not minimally thin in $D$ at $z$ with respect to $X$, then

$$
\int_{E \cap B\left(z,(\kappa R / 4) \wedge r_{0}\right)}\left(\frac{G_{D}\left(x, x_{0}\right)}{G_{D}\left(A_{|x-z|}(z), x_{0}\right)}\right)^{2} \frac{\Psi\left(\delta_{D}(x)^{-1}\right)}{\Psi\left(|x-z|^{-1}\right)} \frac{d x}{|x-z|^{d}}=\infty .
$$

When $D$ is a half-space or when $D$ is a $C^{1,1}$ open set and $X$ is a purely discontinuous unimodal Lévy process, we have an explicit form of the integral test. We first recall the definition of a $C^{1,1}$ open set.

Definition 1.4. An open set $D$ in $\mathbb{R}^{d}$ is said to be a (uniform) $C^{1,1}$ open set if there exist a localization radius $R>0$ and a constant $\Lambda>0$ such that for every $z \in \partial D$, there exist a $C^{1,1}$-function $\psi=\psi_{z}: \mathbb{R}^{d-1} \rightarrow \mathbb{R}$ satisfying $\psi(0)=0$, $\nabla \psi(0)=(0, \ldots, 0),\|\nabla \psi\|_{\infty} \leq \Lambda,|\nabla \psi(x)-\nabla \psi(w)| \leq \Lambda|x-w|$, and an orthonormal coordinate system $C S_{z}$ with its origin at $z$ such that

$$
B(z, R) \cap D=\left\{y=\left(\widetilde{y}, y_{d}\right) \text { in } C S_{z}:|y|<R, y_{d}>\psi(\widetilde{y})\right\} .
$$

The pair $(R, \Lambda)$ is called the characteristics of the $C^{1,1}$ open set $D$.

A $C^{1,1}$ open set $D$ with characteristics $(R, \Lambda)$ can be unbounded and disconnected; the distance between two distinct components of $D$ is at least $R$.

Recall that an open set $D$ is said to satisfy the interior and exterior ball conditions with radius $R_{1}$ if for every $z \in \partial D$, there exist $x \in D$ and $y \in \bar{D}^{c}$ such that $\operatorname{dist}(x, \partial D)=R_{1}, \operatorname{dist}(y, \partial D)=R_{1}, B\left(x, R_{1}\right) \subset D$ and $B\left(y, R_{1}\right) \subset \bar{D}^{c}$. 
It is known (see [3, Definition 2.1 and Lemma 2.2]) that an open set $D$ is a $C^{1,1}$ open set if and only if it satisfies the interior and exterior ball conditions. By taking $R$ smaller if necessary, we will always assume a $C^{1,1}$ open set with characteristics $(R, \Lambda)$ satisfies the interior and exterior ball conditions with radius $R$.

Corollary 1.5. Suppose that either (i) $D$ is a half-space or (ii) $D \subset \mathbb{R}^{d}$ is a $C^{1,1}$ open set and $\gamma=1$ in (1.2). Assume that $E$ is a Borel subset of $D$.

(1) If $E$ is minimally thin in $D$ at $z \in \partial D$ with respect to $X$, then

$$
\int_{E \cap B(z, 1)}|x-z|^{-d} d x<\infty .
$$

(2) Conversely, if $E$ is the union of a subfamily of Whitney cubes of $D$ and is not minimally thin in $D$ at $z \in \partial D$ with respect to $X$, then

$$
\int_{E \cap B(z, 1)}|x-z|^{-d} d x=\infty .
$$

We sometimes write a point $z=\left(z_{1}, \ldots, z_{d}\right) \in \mathbb{R}^{d}$ as $\left(\widetilde{z}, z_{d}\right)$ with $\widetilde{z} \in \mathbb{R}^{d-1}$. Throughout this paper, $\mathbb{H}_{b}$ stands for the set $\left\{x=\left(\widetilde{x}, x_{d}\right) \in \mathbb{R}^{d}: x_{d}>b\right\}$. We will denote the upper half-space $\mathbb{H}_{0}$ by $\mathbb{H}$.

An open set $D$ is said to be half-space-like if, after isometry, there exist two real numbers $b_{1} \leq b_{2}$ such that $\mathbb{H}_{b_{2}} \subset D \subset \mathbb{H}_{b_{1}}$. Without loss of generality, whenever we deal with a half-space-like open set $D$, we will always assume that $\mathbb{H}_{1} \subset D \subset \mathbb{H}$.

Now we state our results on minimal thinness at infinity. In Section 7 we will first extend the main result of 32 to purely discontinuous unimodal Lévy processes so that, for a large class of unbounded open sets including half-space-like open sets, the infinite part of the (minimal) Martin boundary consists of a single point. We call such a point infinity and denote it by $\infty$.

Definition 1.6. We say $\left(\mathcal{E}, \mathcal{F}_{D}\right)$ satisfies the Hardy inequality if there exists $c>0$ such that

$$
\mathcal{E}(v, v) \geq c \int_{D} v^{2}(x) \Psi\left(\delta_{D}(x)^{-1}\right) d x, \quad v \in \mathcal{F}_{D}
$$

Here is the second main result of the paper.

Theorem 1.7. Suppose that (H2) holds and $\gamma=1$ in (1.2). Assume that $D \subset \mathbb{R}^{d}$ is a half-space-like open set and that $\left(\mathcal{E}, \mathcal{F}_{D}\right)$ satisfies the Hardy inequality. Let $E$ be a Borel subset of $D$ and $x_{0}=(\widetilde{0}, 5)$.

(1) If $E$ is minimally thin in $D$ at infinity with respect to $X$, then

$$
\int_{E \cap B(0,1)^{c}}|x|^{d} G_{D}\left(x, x_{0}\right)^{2} \Psi\left(\delta_{D}(x)^{-1}\right) d x<\infty .
$$

(2) Conversely, if $E$ is the union of a subfamily of Whitney cubes of $D$ and is not minimally thin in $D$ at infinity with respect to $X$, then

$$
\int_{E \cap B(0,1)^{c}}|x|^{d} G_{D}\left(x, x_{0}\right)^{2} \Psi\left(\delta_{D}(x)^{-1}\right) d x=\infty .
$$

Again, when $D$ is a half-space-like $C^{1,1}$ open set, we get the following corollary. 
Corollary 1.8. Suppose that (H2) holds and $\gamma=1$ in (1.2). Assume that $D \subset \mathbb{R}^{d}$ is a half-space-like $C^{1,1}$ open set and that $E$ is a Borel subset of $D$.

(1) If $E$ is minimally thin in $D$ at infinity with respect to $X$, then

$$
\int_{E \cap B(0,1)^{c}}|x|^{-d} d x<\infty .
$$

(2) Conversely, if $E$ is the union of a subfamily of Whitney cubes of $D$ and is not minimally thin in $D$ at infinity with respect to $X$, then

$$
\int_{E \cap B(0,1)^{c}}|x|^{-d} d x=\infty .
$$

In order to prove these results we need various potential-theoretic results for the underlying process such as Harnack inequality, boundary Harnack principle, sharp estimates of the Green function and the Martin kernel in $D \subset \mathbb{R}^{d}$, and identification of the Martin boundary of $D$ with the Euclidean boundary. All of these results have been established previously, some of them quite recently, under various conditions on the process $X$ and the open set $D$. The main novelty is that local results for possibly unbounded open sets are obtained only under local conditions on the underlying process $X$, a fact that leads to significant technical difficulties. Therefore we start the paper with three preliminary sections that establish all necessary results. In Section 2 we first recall some previous results from [33]. The main new result is Theorem 2.11, where we prove sharp local estimates of the Green function of $X^{D}$ in case $D$ is a (not necessarily bounded) $\kappa$-fat open set. It is proved in 33 that the finite part of the (minimal) Martin boundary of any $\kappa$-fat set $D$ coincides with the Euclidean boundary of $D$; see [33, Theorem 3.3]. The main result of Section 3 is Theorem 3.2, which gives sharp estimates of the Martin kernel at a finite boundary point of a $\kappa$-fat open set. In Section 4, we assume (H2) holds and extend some results previously known for subordinate Brownian motions.

In Section 5, we will discuss both local results and global results (under the condition (H2)). In that section, we study quasi-additivity of capacity with respect to a Whitney decomposition of $D$. Here we closely follow the method from [1], but use more delicate estimates for the underlying Lévy process. The main novelty here is that we prove local quasi-additivity only under local assumptions on the process $X$; see Proposition 5.11 (1). One of the main ingredients in proving quasiadditivity is a construction of a measure comparable to capacity. Here one needs a Hardy-type inequality for the associated Dirichlet form. We assume the (local) Hardy inequality and at the end of the section give some sufficient conditions for this inequality.

Section 6 is devoted to the proof of Theorem 1.3. After recalling the definition of minimal thinness and giving a general criterion, we establish in Lemma 6.3 the main technical tool for proving the Wiener-type criterion for minimal thinness at a finite boundary point given in Proposition 6.4. Arguments leading from this criterion to its Aikawa version given in Proposition 6.6 are analogous to those of [2, Part II, 7] and rely on the (local) quasi-additivity. The proof of the main Theorem 1.3 is a consequence of Aikawa's criterion and the existence of a comparable measure. In the case when $X$ is a unimodal Lévy process and $D$ a $C^{1,1}$-open set, explicit boundary behaviors of the mean exit times in terms of the distance to the boundary lead to Corollary 1.5, see also the proof of Corollary 6.7, Aikawa's Wiener-criterion for a $C^{1,1}$ open set. 
In Section 7 we assume that $(\mathbf{H 2})$ holds and that $X$ is a unimodal Lévy process. In this section we study minimal thinness at infinity under global assumptions on the underlying process and prove Theorem 1.7. The proofs, although similar to those from the previous section, contain non-trivial modifications (in particular the main technical Lemma 7.2) and are given in full. The starting point of the section is Theorem 7.1, where we extend a recent result from [32] stating that the (minimal) Martin boundary of an open set which is $\kappa$-fat at infinity consists of precisely one point. Besides half-space-like open sets, infinite cones are another example of unbounded sets which are $\kappa$-fat at infinity. Minimal thinness at infinity for infinite cones seems to be more delicate, even in the classical case; see [37, Theorem 1]. That is why we restrict our consideration to half-space-like open sets.

Finally, in Section 8 we study the question of minimal thinness of a set below the graph of a Lipschitz function, both at a finite and infinite boundary point. In case of minimal thinness at a finite boundary point we state in Proposition 8.1 a Burdzy-type criterion which generalizes [28, Theorem 4.4]. As an application of Theorem 1.7 and Corollary 1.8 we prove the main result of Section 8 , a criterion for minimal thinness at infinity under the graph of a Lipschitz function; see Theorem 8.2 .

We conclude this introduction by setting up some notation and conventions. We use ":=" to denote a definition, which is read as "is defined to be"; we denote $a \wedge b:=\min \{a, b\}, a \vee b:=\max \{a, b\}$; we often denote point $z=\left(z_{1}, \ldots, z_{d}\right) \in \mathbb{R}^{d}$ as $\left(\widetilde{z}, z_{d}\right)$ with $\widetilde{z} \in \mathbb{R}^{d-1}$; we denote by $B(x, r)$ the open ball centered at $x \in \mathbb{R}^{d}$ with radius $r>0$; for any two positive functions $f$ and $g, f \asymp g$ means that there is a positive constant $c \geq 1$ so that $c^{-1} g \leq f \leq c g$ on their common domain of definition; for any Borel subset $E \subset \mathbb{R}^{d}$ and $x \in E$, $\operatorname{diam}(E)$ stands for the diameter of $E,|E|$ stands for the Lebesgue measure of $E$ in $\mathbb{R}^{d}$, int $(E)$ stands for the interior of $E$ and $\delta_{E}(x)$ stands for the Euclidean distance between $x$ and $E^{c} ; \mathbb{N}$ is the set of non-negative integers. The values of the constants $R, \delta_{1}, \delta_{2}, \delta_{3}, \delta_{4}, C_{1}, C_{2}, \ldots$ remain the same throughout this paper, while $c, c_{0}, c_{1}, c_{2}, \ldots$ represent constants whose values are unimportant and may change. All constants are positive finite numbers. The labeling of the constants $c_{0}, c_{1}, c_{2}, \ldots$ starts anew in the statement and proof of each result. The dependence of constant $c$ on dimension $d$ is not mentioned explicitly.

\section{Green function estimates}

Throughout this paper, we always assume that $j$ is a strictly positive nonincreasing function on $(0, \infty)$ satisfying (1.1) such that $(\mathbf{H 1})$ holds, and that $X$ is a purely discontinuous symmetric transient Lévy process with Lévy exponent $\Psi_{X}(\xi)$ and a Lévy density $J_{X}$ satisfying (1.2).

As a consequence of (H1), (1.4) and [39, Proposition 28.1] we know that for any $t>0, X_{t}$ has a density $p_{t}(x, y)$ which is smooth. We will use $G(x, y):=$ $\int_{0}^{\infty} p_{t}(x, y) d t$ to denote the Green function of $X$. Since $X$ is a Lévy process, $G(x, y)$ depends on $x-y$ only. Moreover, by the symmetry assumption on $X, G(x, y)=$ $G(y, x)$. For simplicity we use $G(x)$ for $G(x, 0)$.

Given an open set $D \subset \mathbb{R}^{d}$, we define $X_{t}^{D}(\omega)=X_{t}(\omega)$ if $t<\tau_{D}(\omega)$ and $X_{t}^{D}(\omega)=$ $\partial$ if $t \geq \tau_{D}(\omega)$, where $\partial$ is a cemetery state. Throughout this paper, we use the convention that any function $f$ on $D$ is extended to the cemetery state by $f(\partial)=0$. Since $J_{X}$ satisfies the assumption [13, (1.6)], by [13, Theorem 3.1], for every open 
set $D, X_{t}^{D}$ has a Hölder continuous density $p_{D}(t, x, y)$. For any open set $D$ in $\mathbb{R}^{d}$, let $G_{D}(x, y)=\int_{0}^{\infty} p_{D}(t, x, y) d t$ be the Green function of $X^{D}$. The function $G_{D}$ is jointly lower semi-continuous on $D \times D$; see Remark 2.6.

We first recall the definitions of harmonic functions with respect to $X$ and $X^{D}$.

Definition 2.1. Let $D$ be an open subset of $\mathbb{R}^{d}$. A non-negative function $u$ on $\mathbb{R}^{d}$ is said to be (1) harmonic in $D$ with respect to $X$ if $u(x)=\mathbb{E}_{x}\left[u\left(X_{\tau_{B}}\right)\right]$ for each $x \in B$ and every open set $B$ whose closure is a compact subset of $D ;(2)$ regular harmonic in $D$ with respect to $X$ if for each $x \in D, u(x)=\mathbb{E}_{x}\left[u\left(X_{\tau_{D}}\right), \tau_{D}<\infty\right]$.

Definition 2.2. Let $D$ be an open subset of $\mathbb{R}^{d}$. A non-negative function $u$ on $D$ is said to be harmonic with respect to $X^{D}$ if $u(x)=\mathbb{E}_{x}\left[u\left(X_{\tau_{U}}^{D}\right)\right]$ for every $x \in U$ and every open set $U$ whose closure is a compact subset of $D$.

Obviously, if $u$ is harmonic with respect to $X^{D}$, then the function equal to $u$ in $D$ and zero outside $D$ is harmonic with respect to $X$ in $D$. All non-negative functions that are harmonic in $D$ with respect to $X$ are continuous; see 33.

For notational convenience, we define

$$
\Phi(r)=\frac{1}{\Psi^{*}\left(r^{-1}\right)}, \quad r>0 .
$$

The right continuous inverse function of $\Phi$ will be denoted by the usual notation $\Phi^{-1}(r)$.

The following two results are proved in 33 .

Theorem 2.3 ([33, (1.4), (2.1) and Theorem 2.4]). For every $M \geq 1$ there exists a positive constant $C_{1}(M)=C_{1}(M, \Psi, \gamma)$ such that for all $x \in B(0, M)$,

$$
C_{1}(M)^{-1} \frac{\Phi(|x|)}{|x|^{d}} \leq G(x) \leq C_{1}(M) \frac{\Phi(|x|)}{|x|^{d}} .
$$

Lemma 2.4 ([33, (1.4), (2.1) and Lemma 2.7]). For every bounded open set D, the Green function $G_{D}(x, y)$ is finite and continuous off the diagonal of $D \times D$ and there exists $c=c(\operatorname{diam}(D), \Psi, \gamma) \geq 1$ such that for all $x, y \in D$,

$$
G_{D}(x, y) \leq c \frac{\Phi(|x-y|)}{|x-y|^{d}} .
$$

Before we state the interior lower bound on the Green function, we first recall a result from analysis (see [43, Theorem 1, p. 167]): Any open set $D \subset \mathbb{R}^{d}$ is the union of a family $\left\{Q_{j}\right\}_{j \in \mathbb{N}}$ of closed cubes, with sides all parallel to the axes, satisfying the following properties: (i) $\operatorname{int}\left(Q_{j}\right) \cap \operatorname{int}\left(Q_{k}\right)=\emptyset$; (ii) for any $j$, $\operatorname{diam}\left(Q_{j}\right) \leq \operatorname{dist}\left(Q_{j}, \partial D\right) \leq 4 \operatorname{diam}\left(Q_{j}\right)$, where $\operatorname{dist}\left(Q_{j}, \partial D\right)$ denotes the Euclidean distance between $Q_{j}$ and $\partial D$. The family $\left\{Q_{j}\right\}_{j \in \mathbb{N}}$ above is called a Whitney decomposition of $D$ and the $Q_{j}$ 's are called Whitney cubes (of $D$ ). We will use $x_{j}$ to denote the center of the cube $Q_{j}$.

Lemma 2.5. (1) For every $L, T>0$, there exists $c=c(T, L, \Psi, \gamma)>0$ such that for any bounded open set $D$ with $\operatorname{diam}(D) \leq T, x, y \in D$ with $|x-y| \leq L\left(\delta_{D}(x) \wedge \delta_{D}(y)\right)$,

$$
G_{D}(x, y) \geq c \frac{\Phi(|x-y|)}{|x-y|^{d}} .
$$

(2) For every $M \geq 1$, every $L>0$ and any open set $D$, there exists $c=$ $c(M, L, \Psi, \gamma)>0$ such that for every Whitney decomposition $\left\{Q_{j}\right\}$ of $D$, every cube 
$Q_{j}$ such that $\operatorname{diam}\left(Q_{j}\right) \leq M$ and all $x, y \in Q_{j}$ with $|x-y| \leq L\left(\delta_{D}(x) \wedge \delta_{D}(y)\right)$,

$$
G_{D}(x, y) \geq c \frac{\Phi(|x-y|)}{|x-y|^{d}} .
$$

Proof. (1) This part is exactly [33, Lemma 2.8].

(2) Fix $Q_{j}$ with $\operatorname{diam}\left(Q_{j}\right) \leq M$. Recall that $x_{j}$ is the center of $Q_{j}$. Let $\widetilde{D}:=$ $D \cap B\left(x_{j}, 8 M\right)$, so that $\operatorname{diam}(\widetilde{D}) \leq 8 M$. Let $x, y \in Q_{j}$ be such that $|x-y| \leq$ $L\left(\delta_{D}(x) \wedge \delta_{D}(y)\right)$. Since $\operatorname{dist}\left(Q_{j}, \partial D\right) \leq 4 \operatorname{diam}\left(Q_{j}\right) \leq 4 M$, we see that $\delta_{\widetilde{D}}(x)=$ $\delta_{D}(x)$ and $\delta_{\widetilde{D}}(y)=\delta_{D}(y)$. Thus $|x-y| \leq L\left(\delta_{\widetilde{D}}(x) \wedge \delta_{\widetilde{D}}(y)\right)$. From part (1) we conclude that (2.1) holds with $c=c(M, L, \Psi, \gamma)$.

Remark 2.6. By the domain monotonicity of Green functions and Lemmas 2.4 and 2.5(i), one can easily see that the function $G_{D}$ is jointly lower semi-continuous on $D \times D$. In fact, $G_{D}$ is continuous in the extended sense at the diagonal: $\lim _{(x, y) \rightarrow\left(x_{0}, x_{0}\right)} G_{D}(x, y)=G_{D}\left(x_{0}, x_{0}\right)=+\infty$ for any $x_{0} \in D$.

We record a simple consequence of (H1), which we will use several times: There exists a positive constant $c_{1}>0$ such that for all positive $r, s, A$ with $A s \leq r \leq 1$,

$$
\frac{\Phi(r)}{r^{d}} \leq c_{1}\left(A^{-d} \vee A^{-d+2 \delta_{1}}\right) \frac{\Phi(s)}{s^{d}} .
$$

In the remainder of this section, we assume that $D$ is a $\kappa$-fat open set with localization radius $R$. Without loss of generality we may assume that $R \leq \frac{1}{10}$. Recall that for each $z \in \partial D$ and $r \in(0, R), A_{r}(z)$ is a point in $D \cap B(z, r)$ satisfying $B\left(A_{r}(z), \kappa r\right) \subset D \cap B(z, r)$. We also recall that $G_{D}(\cdot, y)$ is regular harmonic in $D \backslash \overline{B(y, \varepsilon)}$ for every $\varepsilon>0$ and vanishes outside $D$.

Lemma 2.7 (Carleson's estimate). There exists a constant $c=c(\Psi, \gamma, \kappa)>1$ such that for every $z \in \partial D, 0<r \leq \kappa R / 4, y \in D \backslash \overline{B(z, 4 r)}$,

$$
G_{D}(x, y) \leq c G_{D}\left(A_{r}(z), y\right), \quad x \in D \cap B(z, r) .
$$

Proof. By the boundary Harnack principle in [33. Theorem 2.3(ii)], it suffices to

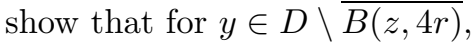

$$
G_{D}\left(x, A_{4 r / \kappa}(z)\right) \leq c_{1} r^{-d} \Phi(r) \leq c_{2} G_{D}\left(A_{r}(z), A_{4 r / \kappa}(z)\right), \quad x \in D \cap B(z, r) .
$$

Since $\left|x-A_{4 r / \kappa}(z)\right| \geq \delta_{D}\left(A_{4 r / \kappa}(z)\right)-\delta_{D}(x) \geq 4 r-r=3 r$, the first inequality in (2.4) follows from Theorem 2.3 and (2.2). On the other hand, since $\mid A_{r}(z)-$ $A_{4 r / \kappa}(z) \mid \leq 8 r \kappa^{-1} \leq 8 \kappa^{-2}\left(\delta_{D}\left(A_{4 r / \kappa}(z)\right) \wedge \delta_{D}\left(A_{r}(z)\right)\right)$, the second inequality in (2.4) follows from Lemma 2.5 (1) and (2.2). The assertion of the lemma follows.

Next we show a localization result for an unbounded $\kappa$-fat set.

Proposition 2.8. Let $D$ be an unbounded $\kappa$-fat set with localization radius $R>0$. There exist $\kappa_{1}=\kappa_{1}(\kappa, R, d) \in(0, \kappa]$ and $R_{1}=R_{1}(\kappa, R, d) \in(0, R]$ such that for every $z_{0} \in \partial D$ there exists a $\kappa_{1}$-open set $D\left(z_{0}\right)$ with localization radius $R_{1}$ satisfying $D \cap B\left(z_{0}, 1\right) \subset D\left(z_{0}\right) \subset D \cap B\left(z_{0}, 2\right)$.

Proof. Recall that $R \leq 1 / 4$ and $\kappa \leq 1 / 2$. We first note that by making $\kappa$ smaller if necessary, we can assume that for any $r \leq R$ and $z \in \bar{D}$, there exists $A_{r}(z) \in D$ such that $B\left(A_{r}(z), \kappa r\right) \subset D \cap B(z, r)$. This means that the $\kappa$-fatness of $D$ at every boundary point $z \in \partial D$ implies that the $\kappa$-fatness property (with possibly smaller $\kappa$ ) 
holds also true at every interior point $z \in D$. In particular, a non-tangential point $A_{r}(z) \in D \cap B(z, r)$ is well defined for every $z \in \bar{D}$.

Let $z_{0} \in \partial D$ and define

$$
D\left(z_{0}\right)=\left(D \cap B\left(z_{0}, 1\right)\right) \cup\left(\bigcup_{z \in \overline{D \cap B\left(z_{0}, 1\right)}} \bigcup_{r \in(0, R]} B\left(A_{r}(z), \kappa r\right)\right) .
$$

Clearly, $D \cap B\left(z_{0}, 1\right) \subset D\left(z_{0}\right) \subset D \cap B\left(z_{0}, 2\right)$. We claim that $D\left(z_{0}\right)$ is $\kappa_{1}$-fat with localization radius $R$, where $\kappa_{1}=\kappa / 32$. Actually, we show that for every $w \in \overline{D\left(z_{0}\right)}$ and every $s \in(0, R]$ there exists $\widetilde{A}_{s}(w) \in D\left(z_{0}\right)$ such that $B\left(\widetilde{A}_{s}(w), \kappa_{1} s\right) \subset D\left(z_{0}\right) \cap$ $B(w, s)$.

Let $w \in \overline{D \cap B\left(z_{0}, 1\right)}$. By the very definition of $D\left(z_{0}\right)$, for each $s \in(0, R]$ we have that $B\left(A_{s}(w), \kappa s\right) \subset D\left(z_{0}\right) \cap B(w, s)$. Hence, we can take $\widetilde{A}_{s}(w)=A_{s}(w)$.

Suppose now that $w \in \overline{B\left(A_{r}(z), \kappa r\right)}$ for some $z \in \overline{D \cap B\left(z_{0}, 1\right)}$ and $r \in(0, R]$. We consider two cases: (i) $s \leq 8 r$, and (ii) $8 r<s \leq R$. In the case $s \leq 8 r$, first note that $2 \kappa_{1} s=(\kappa / 16) s \leq \kappa r / 2$. Consider the line segment connecting $w$ and $A_{r}(z)$ and let $\widetilde{A}_{s}(w)$ be the point on this segment at the distance $2 \kappa_{1} s$ from $w$. If $v \in B\left(\widetilde{A}_{s}(w), \kappa_{1} s\right)$, then

$$
|v-w| \leq\left|v-\widetilde{A}_{s}(w)\right|+\left|\widetilde{A}_{s}(w)-w\right| \leq \kappa_{1} s+2 \kappa_{1} s \leq \frac{3}{32} \kappa s<\frac{s}{2},
$$

and $\left|v-A_{r}(z)\right| \leq\left|w-A_{r}(z)\right| \leq \kappa r$. This proves that $B\left(\widetilde{A}_{s}(w), \kappa_{1} s\right) \subset B\left(A_{r}(z), \kappa r\right)$ $\cap B(w, s / 2) \subset D\left(z_{0}\right) \cap B(w, s / 2)$. If $8 r<s \leq R$, then $B(z, s / 4) \subset B(w, s / 2)$. Indeed, if $v \in B(z, s / 4)$, then

$$
|v-w| \leq|v-z|+\left|z-A_{r}(z)\right|+\left|A_{r}(z)-w\right| \leq \frac{s}{4}+r+\kappa r \leq \frac{s}{4}+\frac{3}{2} r<\frac{s}{2} .
$$

Take $\widetilde{A}_{s}(w)=A_{s / 4}(z)$. Then $B\left(\widetilde{A}_{s}(w), \kappa_{1} s\right) \subset B\left(A_{s / 4}(z), \kappa s / 4\right) \subset D\left(z_{0}\right) \cap$ $B(z, s / 4) \subset D\left(z_{0}\right) \cap B(w, s / 2)$.

Finally, assume that $w$ is in the closure of $\bigcup_{z \in \overline{D \cap B\left(z_{0}, 1\right)}} \bigcup_{r \in(0, R]} B\left(A_{r}(z), \kappa r\right)$. Then there exist sequences $\left(w_{n}\right)_{n \geq 1},\left(z_{n}\right)_{n \geq 1}$ in $\overline{D \cap B\left(z_{0}, 1\right)}$ and $\left(r_{n}\right)_{n \geq 1}$ in $(0, R]$ such that $w_{n} \in B\left(A_{r_{n}}\left(z_{n}\right), \kappa r_{n}\right)$ and $w=\lim _{n} w_{n}$. Let $s \leq R$ and choose $n \geq 1$ so that $\left|w_{n}-w\right| \leq s / 4$. By what has already been proved, there exists $\widetilde{A}_{s}\left(w_{n}\right) \in$ $D\left(z_{0}\right)$ so that $B\left(\widetilde{\widetilde{A}}_{s}\left(w_{n}\right), \kappa_{1} s\right) \subset D\left(z_{0}\right) \cap B\left(w_{n}, s / 2\right)$. Define $\widetilde{A}_{s}(w)=\widetilde{A}_{s}\left(w_{n}\right)$. If $v \in B\left(\widetilde{A}_{s}(w), \kappa_{1} s\right)$, then

$$
|v-w| \leq\left|v-\widetilde{A}_{s}\left(w_{n}\right)\right|+\left|\widetilde{A}_{s}\left(w_{n}\right)-w_{n}\right|+\left|w_{n}-w\right| \leq \kappa_{1} s+\frac{s}{2}+\frac{s}{4}<s .
$$

Thus $B\left(\widetilde{A}_{s}(w), \kappa_{1} s\right) \subset D\left(z_{0}\right) \cap B(w, s)$.

Without loss of generality (by choosing $R$ and $\kappa$ smaller if necessary), we assume in the sequel that $R=R_{1}$ and $\kappa=\kappa_{1}$.

Fix $x_{0} \in D$ with $\kappa R<\delta_{D}\left(x_{0}\right)<R$ (later we will fix a point $z \in \partial D$ and restrict further that $\left.x_{0} \in B(z, R) \cap D\right)$ and set $\varepsilon_{1}:=\frac{\kappa R}{24}$. Define $r(x, y):=\delta_{D}(x) \vee \delta_{D}(y) \vee$ $|x-y|$ for $x, y \in D$, and

$$
\mathcal{B}(x, y):= \begin{cases}\left\{A \in D: \delta_{D}(A)>\frac{\kappa}{2} r(x, y),\right. & \\ |x-A| \vee|y-A|<5 r(x, y)\} & \text { if } r(x, y)<\varepsilon_{1}, \\ \left\{x_{0}\right\} & \text { if } r(x, y) \geq \varepsilon_{1} .\end{cases}
$$


Set $C_{2}:=C_{1}(2) \Phi\left(\frac{\delta_{D}\left(x_{0}\right)}{2}\right)\left(\frac{\delta_{D}\left(x_{0}\right)}{2}\right)^{-d}$, where $C_{1}(2)$ is the constant from Theorem 2.3. By Lemma 2.4 we see that $G_{D}\left(x, x_{0}\right) \leq C_{2}$ for $x \in\left(D \cap B\left(x_{0}, 2\right)\right) \backslash B\left(x_{0}, \frac{\delta_{D}\left(x_{0}\right)}{2}\right)$.

Now we define

$$
g(x):=G_{D}\left(x, x_{0}\right) \wedge C_{2} .
$$

We note that for $y \in D \cap B\left(x_{0}, 2\right)$ with $\delta_{D}(y) \leq 6 \varepsilon_{1}$, we have $g(y)=G_{D}\left(y, x_{0}\right)$, since $6 \varepsilon_{1}<\frac{\delta_{D}\left(x_{0}\right)}{4}$ and thus $\left|y-x_{0}\right| \geq \delta_{D}\left(x_{0}\right)-6 \varepsilon_{1} \geq \frac{\delta_{D}\left(x_{0}\right)}{2}$.

The following lemma follows from [33, Theorem 2.2].

Lemma 2.9. (1) There exists $c=c(\kappa, R, \Psi, \gamma)>1$ such that for every $x \in D \cap$ $B\left(x_{0}, 2\right)$ satisfying $\delta_{D}(x) \geq 2^{-6} \kappa^{3} \varepsilon_{1}$ we have $c^{-1} \leq g(x) \leq c$.

(2) There exists $c=c(\kappa, R, \Psi, \gamma)>0$ such that for every $x, y \in D \cap B\left(x_{0}, 2\right)$, $c^{-1} g\left(A_{1}\right) \leq g\left(A_{2}\right) \leq c g\left(A_{1}\right)$ for every $A_{1}, A_{2} \in \mathcal{B}(x, y)$.

With these preparations, we can prove the following two-sided estimates on the Green functions of bounded $\kappa$-fat open sets (without loss of generality, assuming that $\operatorname{diam}(D) \leq 1$ ), which extend [29, Theorem 1.2]. As we mentioned in [29, Theorem 1.2], with (1.6), Lemmas 2.4 2.5(1), [33, Theorems 2.2 and 2.3] at hand, one can easily adapt the arguments of [7, Proposition 6]. Since these are more or less standard now, we omit the details. (See also the proof of [27, Theorem 6.4].)

Theorem 2.10. If $D$ is a bounded $\kappa$-fat open set with localization radius $R$, then there exists a constant $c=c(\operatorname{diam}(D), R, \kappa, \Psi, \gamma)>1$ such that for every $x, y \in D$ and $A \in \mathcal{B}(x, y)$,

$$
c^{-1} \frac{g(x) g(y) \Phi(|x-y|)}{g(A)^{2}|x-y|^{d}} \leq G_{D}(x, y) \leq c \frac{g(x) g(y) \Phi(|x-y|)}{g(A)^{2}|x-y|^{d}},
$$

where $g$ and $\mathcal{B}(x, y)$ are defined by (2.6) and (2.5) respectively.

In fact, it is the next result, which covers unbounded $\kappa$-fat open sets, that we will use in the following section.

Theorem 2.11. Suppose that $D$ is a $\kappa$-fat open set with localization radius $R$ and $z \in \partial D$. Assume that $x_{0} \in B(z, R) \cap D$ with $\kappa R<\delta_{D}\left(x_{0}\right)<R$. There exists $C_{3}=C_{3}(\Psi, \gamma, R, \kappa)>1$ such that for all $x, y \in B\left(z, 2^{-7} \kappa^{2} R\right) \cap D$ and $A \in \mathcal{B}(x, y)$ it holds that

$$
C_{3}^{-1} \frac{g(x) g(y) \Phi(|x-y|)}{g(A)^{2}|x-y|^{d}} \leq G_{D}(x, y) \leq C_{3} \frac{g(x) g(y) \Phi(|x-y|)}{g(A)^{2}|x-y|^{d}} .
$$

Proof. By Theorem 2.10, we only need to prove the theorem for unbounded $D$.

First, note that $g(\cdot)=G_{D}\left(\cdot, x_{0}\right)$ on $B(z, \kappa R / 4)$. Using Proposition 2.8, we choose a bounded $\kappa$-fat open set $D_{1}$ with localization radius $R$ such that $D \cap B(z, 1) \subset$ $D_{1} \subset D \cap B(z, 2)$. First note that since $\delta_{D_{1}}(x) \vee \delta_{D_{1}}(y)=\delta_{D}(x) \vee \delta_{D}(y) \leq 2^{-7} \kappa^{2} R$, by the boundary Harnack principle in [33, Theorem 2.3(ii)],

$$
\frac{\left(G_{D_{1}}\left(x, x_{0}\right) \wedge C_{2}\right)\left(G_{D_{1}}\left(y, x_{0}\right) \wedge C_{2}\right)}{\left(G_{D_{1}}\left(A, x_{0}\right) \wedge C_{2}\right)^{2}}=\frac{G_{D_{1}}\left(x, x_{0}\right) G_{D_{1}}\left(y, x_{0}\right)}{G_{D_{1}}\left(A, x_{0}\right)^{2}} \asymp \frac{g(x) g(y)}{g(A)^{2}} .
$$

It follows from (2.9) and Theorem 2.10 that

$$
G_{D}(x, y) \geq G_{D_{1}}(x, y) \geq c_{1} \frac{g(x) g(y) \Phi(|x-y|)}{g(A)^{2}|x-y|^{d}} .
$$


On the other hand, by the strong Markov property, the symmetry of $G_{D},(2.9)$ and Theorem 2.10,

$$
\begin{aligned}
G_{D}(x, y) & =G_{D_{1}}(x, y)+\mathbb{E}_{x}\left[G_{D}\left(y, X_{\tau_{D_{1}}}\right)\right] \\
& \leq c_{2} \frac{g(x) g(y) \Phi(|x-y|)}{g(A)^{2}|x-y|^{d}}+\mathbb{E}_{x}\left[G_{D}\left(y, X_{\tau_{D_{1}}}\right)\right] .
\end{aligned}
$$

Thus, since $\inf _{a \leq 1} \Phi(a) a^{-d}>0$, it suffices to show the first inequality below:

$$
\mathbb{E}_{x}\left[G_{D}\left(y, X_{\tau_{D_{1}}}\right)\right] \leq c_{3} \frac{g(x) g(y)}{g(A)^{2}} \leq c_{4} \frac{g(x) g(y) \Phi(|x-y|)}{g(A)^{2}|x-y|^{d}} .
$$

Let $\eta_{0}:=2^{-2} \kappa R$ and $\eta_{1}:=2^{-3} \kappa^{2} R$. Since

$$
|z-A| \leq|x-z|+|A-x| \leq 2^{-7} \kappa^{2} R+5 r(x, y) \leq 11 \cdot 2^{-7} \kappa^{2} R<2^{-3} \kappa^{2} R=\eta_{1},
$$

we have $A \in D \cap B\left(z, \eta_{1}\right)$. Thus by Lemma 2.7 .

$$
G_{D}\left(A, x_{0}\right) \leq c_{5}\left(G_{D}\left(A_{\eta_{0}}(z), x_{0}\right) \wedge G_{D}\left(A_{\eta_{1}}(z), x_{0}\right)\right) .
$$

Applying the boundary Harnack principle in [33, Theorem 2.3(ii)] to $G_{D}(\cdot, w)$ and $G_{D}\left(\cdot, x_{0}\right)$ and using (2.11), we get

$$
\begin{aligned}
\int_{D \backslash D_{1}} G_{D}(y, w) \mathbb{P}_{x}\left(X_{\tau_{D_{1}}} \in d w\right) \\
\quad \leq c_{6} \frac{G_{D}\left(y, x_{0}\right)}{G_{D}\left(A_{\eta_{0}}(z), x_{0}\right)} \int_{D \backslash D_{1}} G_{D}\left(A_{\eta_{0}}(z), w\right) \mathbb{P}_{x}\left(X_{\tau_{D_{1}}} \in d w\right) \\
\quad \leq c_{5} c_{6} \frac{g(y)}{g(A)} \mathbb{E}_{x}\left[G_{D}\left(A_{\eta_{0}}(z), X_{\tau_{D_{1}}}\right)\right] .
\end{aligned}
$$

Using the boundary Harnack principle in [33, Theorem 2.3(ii)] and (2.11) again,

$$
\begin{aligned}
& \mathbb{E}_{x}\left[G_{D}\left(A_{\eta_{0}}(z), X_{\tau_{D_{1}}}\right)\right] \leq c_{7} \frac{G_{D}\left(x, x_{0}\right)}{G_{D}\left(A_{\eta_{1}}(z), x_{0}\right)} \mathbb{E}_{A_{\eta_{1}}(z)}\left[G_{D}\left(A_{\eta_{0}}(z), X_{\tau_{D_{1}}}\right)\right] \\
& \leq c_{7} c_{5} \frac{g(x)}{g(A)} \mathbb{E}_{A_{\eta_{1}}(z)}\left[G_{D}\left(A_{\eta_{0}}(z), X_{\tau_{D_{1}}}\right)\right] .
\end{aligned}
$$

By the strong Markov property and Theorem 2.3 ,

$$
\begin{gathered}
\mathbb{E}_{A_{\eta_{1}}(z)}\left[G_{D}\left(A_{\eta_{0}}(z), X_{\tau_{D_{1}}}\right)\right] \leq \mathbb{E}_{A_{\eta_{1}}(z)}\left[G_{D}\left(A_{\eta_{0}}(z), X_{\tau_{D_{1}}}\right)\right]+G_{D_{1}}\left(A_{\eta_{1}}(z), A_{\eta_{0}}(z)\right) \\
=G_{D}\left(A_{\eta_{1}}(z), A_{\eta_{0}}(z)\right) \leq G\left(A_{\eta_{1}}(z), A_{\eta_{0}}(z)\right) \leq c_{8} \sup _{a \geq \eta_{1}} \frac{\Phi(a)}{a^{d}}:=c_{9}<\infty .
\end{gathered}
$$

Combining (2.12)-(2.14), we have proved the first inequality in (2.10).

We remark in passing that one of the reasons we introduced the function $g$, instead of using only the function $G_{D}\left(\cdot, x_{0}\right)$, is that the function $g$ satisfies the local scale invariant Harnack inequality defined in Definition 5.4 while the function $x \rightarrow G_{D}\left(x, x_{0}\right)$ does not. 


\section{MARTIN KeRnel AND ESTIMATES}

In this section we discuss Martin kernels and their estimates. Let $D$ be an open set in $\mathbb{R}^{d}$. Fix a point $x_{0} \in D$ and define

$$
M_{D}(x, y):=\frac{G_{D}(x, y)}{G_{D}\left(x_{0}, y\right)}, \quad x, y \in D, y \neq x_{0} .
$$

As the process $X^{D}$ satisfies Hypothesis (B) in [34, $D$ has a Martin boundary $\partial_{M} D$ with respect to $X$ and $M_{D}(x, \cdot)$ can be continuously extended to $\partial_{M} D$. The Martin kernel at $z \in \partial_{M} D$ (based at $x_{0} \in D$ ) is denoted by $M_{D}(x, z)$.

Recall that a positive harmonic function $f$ for $X^{D}$ is minimal if, whenever $h$ is a positive harmonic function for $X^{D}$ with $h \leq f$ on $D$, one must have $f=c h$ for some constant $c$.

Definition 3.1. (1) A point $z \in \partial_{M} D$ is called a finite Martin boundary point if there exists a bounded sequence $\left\{w_{n}\right\} \subset D$ converging to $z$ in the Martin topology.

(2) A point $z \in \partial_{M} D$ is called an infinite Martin boundary point if every sequence $\left\{w_{n}\right\} \subset D$ converging to $z$ in the Martin topology is unbounded (in the Euclidean topology).

(3) A point $z \in \partial_{M} D$ is called a minimal Martin boundary point if $M_{D}(\cdot, z)$ is a minimal harmonic function for $X^{D}$. Denote by $\partial_{m} D$ the minimal Martin boundary of $X^{D}$.

In 33. Section 3], we showed that the finite part of the (minimal) Martin boundary of any $\kappa$-fat set $D$ coincides with the Euclidean boundary of $D$; see $[33$, Theorem $3.3]$.

Using Theorem 2.11, we get the following Martin kernel estimates. Recall that $g$ is defined by (2.6).

Theorem 3.2. Suppose that $D$ is a $\kappa$-fat open set with localization radius $R$ and $z \in \partial D$. Assume that $x_{0} \in B(z, R) \cap D$ with $\kappa R<\delta_{D}\left(x_{0}\right)<R$. There exists a constant $C_{4}>0$ such that for all $x \in B\left(z, 2^{-7} \kappa^{2} R\right) \cap D$,

$$
C_{4}^{-1} \frac{g(x) \Phi(|x-z|)}{g\left(A_{|x-z|}(z)\right)^{2}|x-z|^{d}} \leq M_{D}(x, z) \leq C_{4} \frac{g(x) \Phi(|x-z|)}{g\left(A_{|x-z|}(z)\right)^{2}|x-z|^{d}} .
$$

Proof. Fix a point $x_{1} \in B\left(z, 2^{-7} \kappa^{2} R\right) \cap D$. First we deal with the Martin kernel $M_{D}^{x_{1}}$ based at $x_{1}$. Since

$$
\delta_{D}(x) \vee \delta_{D}(y) \vee|x-y| \rightarrow|x-z| \quad \text { and } \quad \delta_{D}\left(x_{0}\right) \vee \delta_{D}(y) \vee\left|x_{1}-y\right| \rightarrow\left|x_{1}-z\right|
$$

as $y \rightarrow z$, applying Theorem 2.11 to $(x, y)$ and $\left(x_{1}, y\right)$ respectively, we get

$$
M_{D}^{x_{1}}(x, z) \asymp \frac{g(x) \Phi(|x-y|)\left|x_{1}-z\right|^{d}}{g\left(x_{1}\right) \Phi\left(\left|x_{1}-z\right|\right) g\left(A_{|x-z|}(z)\right)^{2}|x-z|^{d}} \asymp \frac{g(x) \Phi(|x-z|)}{g\left(A_{|x-z|}(z)\right)^{2}|x-z|^{d}} .
$$

The assertion of the theorem follows immediately from the relationship $M_{D}(x, \cdot)=$ $M_{D}^{x_{1}}(x, \cdot) M_{D}\left(x_{1}, \cdot\right)$.

\section{Some Results under (H1) ANd (H2)}

In this section we assume that (H2) also holds. We will extend some known results and prove a new Green function estimate. Our approach is heavily based on some recent results in $[8,9,12,14,15,33$. Note that $(\mathbf{H 2})$ implies transience of the process $X$ (since we are always assuming $d \geq 2$ ). 
First note that if both (H1) and (H2) hold, there exist $a_{5}, a_{6}>0$ such that

$$
a_{5}\left(\frac{R}{r}\right)^{2\left(\delta_{1} \wedge \delta_{3}\right)} \leq \frac{\Psi(R)}{\Psi(r)} \leq a_{6}\left(\frac{R}{r}\right)^{2\left(\delta_{2} \vee \delta_{4}\right)}, \quad a>0,0<r<R<\infty ;
$$

cf. [31, (2.6)].

It follows from [8,15, that there exists a constant $C_{5}>1$ such that

$$
C_{5}^{-1} \frac{\Phi(|x|)}{|x|^{d}} \leq G(x) \leq C_{5} \frac{\Phi(|x|)}{|x|^{d}}, \quad \text { for all } x \in \mathbb{R}^{d},
$$

and

$$
C_{5}^{-1} \frac{1}{|x|^{d} \Phi(|x|)} \leq J_{X}(x) \leq C_{5} \frac{1}{|x|^{d} \Phi(|x|)}, \quad \text { for all } x \in \mathbb{R}^{d}
$$

The next result is proved in a more general setting in [12, Section 3]. In fact, one can also follow the proofs in [14, Section 3] to see that all the arguments of [14, Section 3] with $T=\infty$ go through using the (global) parabolic Harnack inequality in [15], (1.1), (1.2), (4.1), (4.3) and the semigroup property. Thus by following the arguments in [14, Section 3] line by line, one can also prove the next proposition. We omit the details.

Proposition 4.1. Suppose that (H2) holds. Let a be a positive constant. There exists $c=c(a, \Psi, \gamma)>0$ such that for any open set $D$,

$$
p_{D}(t, x, y) \geq c\left(\left(\Phi^{-1}(t)\right)^{-d} \wedge t J_{X}(x-y)\right)
$$

for every $(t, x, y) \in(0, \infty) \times D \times D$ with $\delta_{D}(x) \wedge \delta_{D}(y) \geq a \Phi^{-1}(t)$.

Using (4.3) and Proposition 4.1, the proof of the next lemma is almost identical to that of [33, Lemma 2.8]. We omit the details.

Lemma 4.2. Suppose that (H2) holds. For every $L>0$ and every open set $D$, there exists $c=c(L, \Psi, \gamma)>0$ such that for every $|x-y| \leq L\left(\delta_{D}(x) \wedge \delta_{D}(y)\right)$,

$$
G_{D}(x, y) \geq c \frac{\Phi(|x-y|)}{|x-y|^{d}} .
$$

We now recall the following (global) scale invariant boundary Harnack inequality from 33 that will be used in Section 7.

Theorem 4.3 ([33, Theorem 2.3(i)(ii) and Remark 2.1]). Suppose that (H2) holds. There exists $c=c(\Psi, \gamma) \geq 1$ such that the following hold for all $r>0$.

(i) For every $z_{0} \in \mathbb{R}^{d}$, every open set $U \subset B\left(z_{0}, r\right)$ and for any non-negative function $u$ in $\mathbb{R}^{d}$ which is regular harmonic in $U$ with respect to $X$ and vanishes a.e. in $U^{c} \cap B\left(z_{0}, r\right)$, it holds that

$$
\begin{aligned}
c^{-1} \mathbb{E}_{x}\left[\tau_{U}\right] \int_{B\left(z_{0}, r / 2\right)^{c}} j\left(\left|y-z_{0}\right|\right) u(y) d y & \leq u(x) \\
& \leq c \mathbb{E}_{x}\left[\tau_{U}\right] \int_{B\left(z_{0}, r / 2\right)^{c}} j\left(\left|y-z_{0}\right|\right) u(y) d y
\end{aligned}
$$

for every $x \in U \cap B\left(z_{0}, r / 2\right)$. 
(ii) For every $z_{0} \in \mathbb{R}^{d}$, every open set $D \subset \mathbb{R}^{d}$ and any non-negative functions $u, v$ in $\mathbb{R}^{d}$ which are regular harmonic in $D \cap B\left(z_{0}, r\right)$ with respect to $X$ and vanish a.e. in $D^{c} \cap B\left(z_{0}, r\right)$, we have

$$
\frac{u(x)}{v(x)} \leq c^{4} \frac{u(y)}{v(y)}, \quad \text { for all } x, y \in D \cap B\left(z_{0}, r / 2\right) \text {. }
$$

The next theorem is a consequence of [9, Theorem 4.1 and Corollary 4.5] and Theorem $4.3(\mathrm{i})$.

Theorem 4.4. Suppose that (H2) holds and $\gamma=1$ in (1.2). There exists $c=$ $c(\Psi)>0$ such that for every $C^{1,1}$ with characteristics $(R, \Lambda), r \in(0, R], z \in \partial D$ and any non-negative function $u$ in $\mathbb{R}^{d}$ which is harmonic in $D \cap B(z, r)$ with respect to $X$ and vanishes continuously on $D^{c} \cap B(z, r)$, we have

$$
\frac{u(x)}{u(y)} \leq c \sqrt{\frac{\Phi\left(\delta_{D}(x)\right)}{\Phi\left(\delta_{D}(y)\right)}}, \quad \text { for every } x, y \in D \cap B\left(z, \frac{r}{2}\right) .
$$

\section{QUASI-ADDITIVITY OF CAPACITY}

In this section we will prove two types of results, local and global. For the global case, we assume that (H2) holds. We always state the condition (H2) explicitly when we deal with the global case.

Let Cap denote the capacity with respect to $X$ and Cap $_{D}$ the capacity with respect to the killed process $X^{D}$. The goal of this section is to prove that $\operatorname{Cap}_{D}$ is (locally) quasi-additive with respect to Whitney decompositions of $D$.

We first revisit [42, Section 5.4.1] and extend [42, Proposition 5.55]. Recall that $G(x, y)$ (and $\left.G_{D}(x, y)\right)$ is the Green function of $X$ (and $X^{D}$, respectively), and let $G \mu(x)=\int G(x, y) \mu(d y)$ and $G_{D} \mu(x)=\int_{D} G(x, y) \mu(d y)$.

For any compact subset $K$ of $\mathbb{R}^{d}$, let $\mathcal{P}_{K}$ be the set of probability measures supported by $K$. Define

$$
e(K):=\inf _{\mu \in \mathcal{P}_{K}} \int G \mu(x) \mu(d x) .
$$

Since the kernel $G$ satisfies the Maria-Frostman maximum principle saying that $\sup _{\mathbb{R}^{d}} G \mu=\sup _{\operatorname{Supp}(\mu)} G \mu$ (see, for example, Theorem 5.2.2 in [16]), it follows from (23], p. 159) that for any compact subset $K$ of $\mathbb{R}^{d}$,

$$
\operatorname{Cap}(K)=\frac{1}{\inf _{\mu \in \mathcal{P}_{K}} \sup _{x \in \operatorname{Supp}(\mu)} G \mu(x)}=\frac{1}{e(K)} .
$$

Furthermore, the infimum is attained at the capacitary measure $\mu_{K}$.

Using (5.1), the proof of the next lemma is the same as that of [42, Lemma 5.54].

Lemma 5.1. Let $K$ be a compact subset of $\mathbb{R}^{d}$. For any probability measure $\mu$ on $K$, it holds that

$$
\inf _{x \in \operatorname{Supp}(\mu)} G \mu(x) \leq e(K) \leq \sup _{x \in \operatorname{Supp}(\mu)} G \mu(x) .
$$

Proposition 5.2. There exist positive constants $c_{1}<c_{2}$ such that

$$
c_{1} \frac{r^{d}}{\Phi(r)} \leq \operatorname{Cap}(\overline{B(0, r)}) \leq c_{2} \frac{r^{d}}{\Phi(r)} \quad \text { for every } r \in(0,1] .
$$

Furthermore, if we assume (H2), (5.3) holds for all $r>0$. 
Proof. We first consider the first claim. By (5.1) it suffices to show that

$$
c_{1} \frac{\Phi(r)}{r^{d}} \leq e(\overline{B(0, r)}) \leq c_{2} \frac{\Phi(r)}{r^{d}} \quad \text { for every } r \in(0,1] .
$$

For every $w \in \overline{B(0, r)}$, consider the intersection of $B(0, r)$ and $B(w, r)$. This intersection contains the intersection of $B(w, r)$ and the cone with vertex $w$ of aperture equal to $\pi / 3$ pointing towards the origin. Let $C(w)$ be the latter intersection. Then by Theorem 2.3 .

$$
\begin{aligned}
\int_{B(0, r)} G(w, y) d y & \geq \int_{C(w)} G(w, y) d y \geq c_{1} \int_{B(w, r)} \frac{\Phi(|w-y|)}{|w-y|^{d}} d y \\
& =c_{1} \int_{B(0, r)} \frac{\Phi(|y|)}{|y|^{d}} d y .
\end{aligned}
$$

Thus by (H1),

$$
\inf _{w \in \overline{B(0, r)}} \int_{B(0, r)} G(w, y) d y \geq c_{1} \int_{B(0, r)} \frac{\Phi(|y|)}{|y|^{d}} d y \geq c_{2} \Phi(r) .
$$

On the other hand, Theorem 2.3 and (H1) also give

$$
\begin{aligned}
\sup _{w \in \overline{B(0, r)}} \int_{B(0, r)} G(w, y) d y & \leq c_{3} \sup _{w \in \overline{B(0, r)}} \int_{B(0, r)} \frac{\Phi(|w-y|)}{|w-y|^{d}} d y \\
& \leq c_{3} \int_{B(0,2 r)} \frac{\Phi(|y|)}{|y|^{d}} d y \leq c_{4} \Phi(r) .
\end{aligned}
$$

By applying Lemma 5.1 with the normalized Lebesgue measure on $B(0, r)$, the proposition now follows from (5.2), (5.5) and (5.6).

If we assume (H2), we use (4.1) and (4.2) instead of Theorem 2.3 and (H1) respectively. Then we get (5.4) for all $r>0$ by the same argument.

For any open set $D \subset \mathbb{R}^{d}$ let $\mathcal{S}(D)$ denote the collection of all excessive functions with respect to $X^{D}$ and let $\mathcal{S}^{c}(D)$ be the family of positive functions in $\mathcal{S}(D)$ which are continuous in the extended sense. Recall that positive harmonic functions with respect to $X^{D}$ are in $\mathcal{S}^{c}(D)$. For any $u \in \mathcal{S}(D)$ and $E \subset D$, the reduced function of $u$ relative to $E$ in $D$ is defined by

$$
R_{u}^{E}(x)=\inf \{v(x): v \in \mathcal{S}(D) \text { and } v \geq u \text { on } E\}, \quad x \in \mathbb{R}^{d} .
$$

The lower semi-continuous regularization $\widehat{R}_{u}^{E}$ of $R_{u}^{E}$ is called the balayage of $u$ relative to $E$ in $D$. Since the process $X^{D}$ has a continuous transition density $p_{D}(t, x, y)$, its semi-group is strongly Feller. Thus it follows easily from [6, Proposition V.2.2] that the cone of excessive functions $\mathcal{S}(D)$ is a balayage space in the sense of [6].

Given $u \in \mathcal{S}^{c}(D)$, define a kernel $k_{u}: D \times D \rightarrow[0, \infty]$ by

$$
k_{u}(x, y):=\frac{G_{D}(x, y)}{u(x) u(y)}, \quad x, y \in D
$$

Note that $k_{u}(x, y)$ is jointly lower semi-continuous on $D \times D$ by Remark 2.6 and the assumptions that $u$ is positive and continuous in the extended sense. For a 
measure $\lambda$ on $D$ let $\lambda_{u}(d y):=\lambda(d y) / u(y)$. Then

$$
\begin{aligned}
k_{u} \lambda(x) & :=\int_{D} k_{u}(x, y) \lambda(d y)=\int_{D} \frac{G_{D}(x, y)}{u(x) u(y)} \lambda(d y) \\
& =\frac{1}{u(x)} \int_{D} G_{D}(x, y) \frac{\lambda(d y)}{u(y)}=\frac{1}{u(x)} G_{D} \lambda_{u}(d y) .
\end{aligned}
$$

We define a capacity with respect to the kernel $k_{u}$ as follows:

$$
\mathcal{C}_{u}(E):=\inf \left\{\|\lambda\|: k_{u} \lambda \geq 1 \text { on } E\right\}, \quad E \subset D,
$$

where $\|\lambda\|$ denotes the total mass of the measure $\lambda$ on $D$. The following dual representation of the capacity of compact sets can be found in [24, Théorème 1.1]:

$$
\mathcal{C}_{u}(K)=\sup \left\{\mu(K): \mu(D \backslash K)=0, k_{u} \mu \leq 1 \text { on } D\right\} .
$$

For a compact set $K \subset D$, consider the balayage $\widehat{R}_{u}^{K}$. Being a potential, $\widehat{R}_{u}^{K}=$ $G_{D} \lambda^{K, u}$ for a measure $\lambda^{K, u}$ supported in $K$. Define the Green energy of $K$ (with respect to $u$ ) as

$$
\begin{aligned}
\gamma_{u}(K) & :=\int_{D} \int_{D} G_{D}(x, y) \lambda^{K, u}(d x) \lambda^{K, u}(d y) \\
& =\int_{D} G_{D} \lambda^{K, u}(x) \lambda^{K, u}(d x)=\mathcal{E}\left(G_{D} \lambda^{K, u}, G_{D} \lambda^{K, u}\right) .
\end{aligned}
$$

As usual, this definition of energy is extended first to open and then to Borel subsets of $D$. The following proposition relates the energy $\gamma_{u}$ with the capacity $\mathcal{C}_{u}$.

Proposition 5.3. For all Borel subsets $E \subset D$ it holds that $\gamma_{u}(E)=\mathcal{C}_{u}(E)$.

Proof. Clearly, it suffices to prove the proposition for compact subsets $K$ of $D$. Note first that

$$
\begin{aligned}
\sup \{ & \left.\mu(K): \mu(D \backslash K)=0, k_{u} \mu \leq 1 \text { on } D\right\} \\
& =\sup \left\{\mu(K): \mu(D \backslash K)=0, G_{D} \mu_{u} \leq u \text { on } D\right\} \\
& =\sup \left\{\int_{D} u(y) \lambda(d y), \lambda(D \backslash K)=0, G_{D} \lambda \leq u \text { on } D\right\} .
\end{aligned}
$$

Since $\lambda^{K, u}(D \backslash K)=0$ and $G_{D} \lambda^{K, u}=\widehat{R}_{u}^{K} \leq u$ on $D$, we conclude from the above and (5.9) that

$$
\mathcal{C}_{u}(K) \geq \int_{K} u(y) \lambda^{K, u}(d y) \geq \int_{K} G_{D} \lambda^{K, u}(y) \lambda^{K, u}(d y)=\gamma_{u}(K) .
$$

Conversely, $G_{D} \lambda^{K, u}=\widehat{R}_{u}^{K}=u$ on $K$; hence by the definition of $\mathcal{C}_{u}(K)$ we have

$$
\mathcal{C}_{u}(K) \leq \int_{D} u(y) \lambda^{K, u}(d y)=\int_{K} G_{D} \lambda^{K, u}(y) \lambda^{K, u}(d y)=\gamma_{u}(K) .
$$

Note that in case $u \equiv 1, \gamma_{1}(E)=\mathcal{C}_{1}(E)=\operatorname{Cap}_{D}(E)$.

Let $\left\{Q_{j}\right\}_{j \geq 1}$ be a Whitney decomposition of $D$. Recall that $x_{j}$ is the center of $Q_{j}$. For each $Q_{j}$, let $Q_{j}^{*}$ denote the interior of the double of $Q_{j}$. Then $\left\{Q_{j}, Q_{j}^{*}\right\}$ is a quasi-disjoint decomposition of $D$ in the sense of [2, pp. 146-147]. 
Definition 5.4. (1) A kernel $k: D \times D \rightarrow[0,+\infty]$ is said to satisfy the local Harnack property with respect to $\left\{Q_{j}, Q_{j}^{*}\right\}$ if

$$
k(x, y) \asymp k\left(x^{\prime}, y\right) \text {, for all } x, x^{\prime} \in Q_{j} \text { and all } y \in D \backslash Q_{j}^{*},
$$

for all cubes $Q_{j}$ whose diameter is less than $r_{1}$ for some $r_{1}>0$ (with constants independent of the cubes).

(2) A kernel $k: D \times D \rightarrow[0,+\infty]$ is said to satisfy the Harnack property with respect to $\left\{Q_{j}, Q_{j}^{*}\right\}$ if (5.10) holds for all cubes $Q_{j}$.

Definition 5.5. (1) A function $u: D \rightarrow(0, \infty)$ is said to satisfy the local scale invariant Harnack inequality with respect to $\left\{Q_{j}\right\}$ if for some $r_{1}>0$ there exists $c_{1}=c_{1}\left(r_{1}\right)>0$ such that

$$
\sup _{Q_{j}} u \leq c_{1} \inf _{Q_{j}} u, \quad \text { for all } Q_{j} \text { with } \operatorname{diam}\left(Q_{j}\right)<r_{1} .
$$

(2) A function $u: D \rightarrow(0, \infty)$ is said to satisfy the scale invariant Harnack inequality with respect to $\left\{Q_{j}\right\}$ if there exists $c_{2}>0$ such that

$$
\sup _{Q_{j}} u \leq c_{2} \inf _{Q_{j}} u, \text { for all } Q_{j} .
$$

Lemma 5.6. If $u \in \mathcal{S}^{c}(D)$ satisfies the (local) scale invariant Harnack inequality with respect to $\left\{Q_{j}\right\}$, then the kernel $k_{u}$ satisfies the (local) Harnack property with respect to $\left\{Q_{j}, Q_{j}^{*}\right\}$.

Proof. Note that for all $Q_{j}$ with $\operatorname{diam}\left(Q_{j}\right)<r_{1}$ (respectively for all $Q_{j}$ ), the function $G_{D}(\cdot, y)$ is regular harmonic in $Q_{j}$ for every $y \in D \backslash Q_{j}^{*}$. Together with the assumption that $u$ satisfies the (local) scale invariant Harnack inequality, this proves the claim.

Typical examples of positive continuous excessive functions $u$ that satisfy the local scale invariant Harnack inequality are functions $u \equiv 1$ and $u=G_{D}\left(\cdot, x_{0}\right) \wedge c$ with $x_{0} \in D$ and $c>0$ fixed. Similarly, if $X$ satisfies (H2), the scale invariant Harnack inequality holds for the same functions; see [15, Theorem 4.12].

We now record two lemmas.

Lemma 5.7. (1) There exists a constant $c=c\left(\Psi, \gamma, r_{1}\right) \in(0,1)$ such that

$$
c \operatorname{Cap}_{D}\left(Q_{j}\right) \leq \operatorname{Cap}\left(Q_{j}\right) \leq \operatorname{Cap}_{D}\left(Q_{j}\right)
$$

for all Whitney cubes whose diameter is less than $r_{1}$.

(2) Suppose that $(\mathbf{H 2})$ holds. Then there exists a constant $c=c(\Psi, \gamma) \in(0,1)$ such that (5.13) holds for all Whitney cubes.

Proof. (1) By (5.9) and Proposition 5.3 we have that for every compact set $K \subset D$,

$$
\operatorname{Cap}_{D}(K)=\sup \left\{\mu(K): \operatorname{supp}(\mu) \subset K, G_{D} \mu \leq 1 \text { on } D\right\} \text {. }
$$

If $\operatorname{supp}(\mu) \subset K$ and $G \mu \leq 1$ on $\mathbb{R}^{d}$, then clearly $G_{D} \mu \leq 1$ on $D$. This implies that $\operatorname{Cap}(K) \leq \operatorname{Cap}_{D}(K)$ for all compact subsets $K \subset D$, in particular for each Whitney cube $Q_{j}$.

If $x, y \in Q_{j}$, then $|x-y| \leq \operatorname{diam}\left(Q_{j}\right)<r_{1}$ and $|x-y| \leq \operatorname{diam}\left(Q_{j}\right) \leq$ $\operatorname{dist}\left(Q_{j}, \partial D\right) \leq \delta_{D}(x) \wedge \delta_{D}(y)$. Thus by Lemma 2.5(2) and Theorem 2.3 there exists $c \in(0,1)$ such that for all $Q_{j}$ of diameter less than $r_{1}$ it holds that

$$
G_{D}(x, y) \geq c G(x, y), \quad x, y \in Q_{j} .
$$


Let $\mu$ be the capacitary measure for $Q_{j}$ (with respect to $X^{D}$ ), i.e., $\mu\left(Q_{j}\right)=$ $\operatorname{Cap}_{D}\left(Q_{j}\right)$. Then by (5.14) for every $x \in Q_{j}$ we have

$$
1 \geq G_{D} \mu(x)=\int_{Q_{j}} G_{D}(x, y) \mu(d y) \geq \int_{Q_{j}} c G(x, y) \mu(d y)=G(c \mu)(x) .
$$

By the maximum principle it follows that $G(c \mu) \leq 1$ everywhere on $\mathbb{R}^{d}$. Hence, $\operatorname{Cap}\left(Q_{j}\right) \geq(c \mu)\left(Q_{j}\right)=c \operatorname{Cap}_{D}\left(Q_{j}\right)$.

(2) The proof is analogous: We use Lemma 4.2 instead of Lemma 2.5 (2), and (4.3) instead of Theorem 2.3 .

Lemma 5.8. (1) Suppose that $u \in \mathcal{S}^{c}(D)$ is a function satisfying the local scale invariant Harnack inequality (5.11). Then for every $Q_{j}$ of diameter less than $r_{1}$ and every $E \subset Q_{j}$ it holds that

$$
\gamma_{u}(E) \asymp u\left(x_{j}\right)^{2} \operatorname{Cap}_{D}(E) .
$$

(2) Suppose that (H2) holds and let $u \in \mathcal{S}^{c}(D)$ be a function satisfying the scale invariant Harnack inequality (5.12). Then (5.15) holds for every $Q_{j}$ and every $E \subset Q_{j}$

Proof. (1) It suffices to prove (5.15) for compact subsets $K \subset E \subset Q_{j}$ and for $Q_{j}$ of diameter less than $r_{1}$. Since $u$ satisfies the local Harnack property for $\left\{Q_{j}, Q_{j}^{*}\right\}$, we have $u \asymp u\left(x_{j}\right)$ on $Q_{j}$. Hence $\widehat{R}_{u}^{K} \asymp u\left(x_{j}\right) \widehat{R}_{1}^{K}$, implying $G_{D} \lambda^{K, u} \asymp u\left(x_{j}\right) G_{D} \lambda^{K, 1}$ (everywhere on $D$ ). Therefore,

$$
\begin{aligned}
\gamma_{u}(K) & =\int_{K} G_{D} \lambda^{K, u}(x) \lambda^{K, u}(d x) \asymp u\left(x_{j}\right) \int_{K} G_{D} \lambda^{K, 1}(x) \lambda^{K, u}(d x) \\
& =u\left(x_{j}\right) \int_{K} G_{D} \lambda^{K, u}(x) \lambda^{K, 1}(d x) \asymp u\left(x_{j}\right)^{2} \int_{K} \lambda^{K, 1}(d x)=u\left(x_{j}\right)^{2} \operatorname{Cap}_{D}(K) .
\end{aligned}
$$

(2) The proof is analogous to the proof of (1).

Definition 5.9. Let $\left\{Q_{j}\right\}$ be a Whitney decomposition of $D$.

(1) A Borel measure $\sigma$ on $D$ is locally comparable to the capacity $\mathcal{C}_{u}$ with respect to $\left\{Q_{j}\right\}$ at $z \in \partial D$ if there exists $r_{1}, c_{1}>0$ such that

$$
\begin{array}{ll}
\sigma\left(Q_{j}\right) \asymp \mathcal{C}_{u}\left(Q_{j}\right), & \text { for all } Q_{j} \text { with } Q_{j} \cap B\left(z, r_{1}\right) \neq \emptyset, \\
\sigma(E) \leq c_{1} \mathcal{C}_{u}(E), & \text { for all Borel } E \subset D \cap B\left(z, 2 r_{1}\right) .
\end{array}
$$

(2) A Borel measure $\sigma$ on $D$ is comparable to the capacity $\mathcal{C}_{u}$ with respect to $\left\{Q_{j}\right\}$ if there exists $c_{2}>0$ such that $\sigma\left(Q_{j}\right) \asymp \mathcal{C}_{u}\left(Q_{j}\right)$ for all $Q_{j}$, and $\sigma(E) \leq c_{2} \mathcal{C}_{u}(E)$ for all Borel $E \subset D$.

In order to construct a comparable measure we need (local) Hardy's inequalities. Recall that the local Hardy inequality at $z \in \partial D$ and the Hardy inequality were introduced in Definition [1.1 and Definition [1.6 respectively.

Define

$$
\sigma_{u}(E):=\int_{E} u(x)^{2} \Psi\left(\delta_{D}(x)^{-1}\right) d x, \quad E \subset D .
$$

Proposition 5.10. (1) If $\left(\mathcal{E}, \mathcal{F}_{D}\right)$ satisfies the local Hardy inequality at $z \in \partial D$, then for any Whitney decomposition $\left\{Q_{j}\right\}$ of $D$ and any $u \in \mathcal{S}^{c}(D)$ satisfying the local scale invariant Harnack inequality for $\left\{Q_{j}\right\}, \sigma_{u}$ is locally comparable to the capacity $\mathcal{C}_{u}$ with respect to $\left\{Q_{j}\right\}$ at $z$. 
(2) Suppose that $(\mathbf{H 2})$ holds and that $\left(\mathcal{E}, \mathcal{F}_{D}\right)$ satisfies the Hardy inequality. Then for any Whitney decomposition $\left\{Q_{j}\right\}$ of $D$ and any $u \in \mathcal{S}^{c}(D)$ satisfying the scale invariant Harnack inequality for $\left\{Q_{j}\right\}, \sigma_{u}$ is comparable to the capacity $\mathcal{C}_{u}$ with respect to $\left\{Q_{j}\right\}$.

Proof. (1) Fix $z \in \partial D$ and let $r_{1}=r_{0} / 2$ where $r_{0}$ is the constant in Definition 1.1. Since $u$ satisfies the local scale invariant Harnack inequality for $\left\{Q_{j}\right\}$, we have $u \asymp u\left(x_{j}\right)$ whenever the diameter of $Q_{j}$ is less than $r_{1}$. By Lemma 5.8(1) we have that $\gamma_{u}\left(Q_{j}\right) \asymp u\left(x_{j}\right)^{2} \operatorname{Cap}_{D}\left(Q_{j}\right)$ whenever the diameter of $Q_{j}$ is less than $r_{1}$. On the other hand,

$$
\sigma_{u}\left(Q_{j}\right)=\int_{Q_{j}} u(x)^{2} \Psi\left(\delta_{D}(x)^{-1}\right) d x \asymp u\left(x_{j}\right)^{2} \Psi\left(\left(\operatorname{diam}\left(Q_{j}\right)\right)^{-1}\right)\left|Q_{j}\right| .
$$

Lemma 5.7(1) and Proposition 5.2 imply that

$$
\begin{aligned}
\operatorname{Cap}_{D}\left(Q_{j}\right) & \asymp \operatorname{Cap}\left(Q_{j}\right) \asymp \frac{\left(\operatorname{diam}\left(Q_{j}\right)\right)^{d}}{\Phi\left(\operatorname{diam}\left(Q_{j}\right)\right)} \\
& \asymp \Psi\left(\left(\operatorname{diam}\left(Q_{j}\right)\right)^{-1}\right)\left|Q_{j}\right| \quad \text { for all } Q_{j} \text { with } Q_{j} \cap B\left(z ; r_{1}\right) \neq \emptyset .
\end{aligned}
$$

Thus, $\gamma_{u}\left(Q_{j}\right) \asymp \sigma_{u}\left(Q_{j}\right)$.

Using the local Hardy inequality, for any Borel subset $E \subset D \cap B\left(z, 2 r_{1}\right)$ and compact $K \subset E$,

$$
\begin{aligned}
\gamma_{u}(E) & \geq \gamma_{u}(K)=\mathcal{E}\left(G_{D} \lambda^{K, u}, G_{D} \lambda^{K, u}\right) \geq c_{1} \int_{K}\left(G_{D} \lambda^{K, u}\right)(x)^{2} \Psi\left(\delta_{D}(x)^{-1}\right) d x \\
& =c_{1} \int_{K} u(x)^{2} \Psi\left(\delta_{D}(x)^{-1}\right) d x=c_{1} \sigma_{u}(K) .
\end{aligned}
$$

This proves that $\gamma_{u}(E) \geq c_{1} \sigma_{u}(E)$.

Part (2) is proved analogously.

Now we can repeat the argument in the proof of [2, Theorem 7.1.3] and conclude the following.

Proposition 5.11. (1) If $\left(\mathcal{E}, \mathcal{F}_{D}\right)$ satisfies the local Hardy inequality at $z \in \partial D$, then for any Whitney decomposition $\left\{Q_{j}\right\}$ of $D$ and any $u \in \mathcal{S}^{c}(D)$ satisfying the local scale invariant Harnack inequality for $\left\{Q_{j}\right\}$, the Green energy $\gamma_{u}$ is locally quasi-additive with respect to $\left\{Q_{j}\right\}$ at $z$ : There exists a positive constant $r_{1}>0$ such that

$$
\gamma_{u}(E) \asymp \sum_{j \geq 1} \gamma_{u}\left(E \cap Q_{j}\right) \quad \text { for all Borel } E \subset D \cap B\left(z, r_{1}\right) .
$$

(2) Suppose that $(\mathbf{H 2})$ holds. If $\left(\mathcal{E}, \mathcal{F}_{D}\right)$ satisfies the Hardy inequality, then for any Whitney decomposition $\left\{Q_{j}\right\}$ of $D$ and any $u \in \mathcal{S}^{c}(D)$ satisfying the scale invariant Harnack inequality for $\left\{Q_{j}\right\}$, the Green energy $\gamma_{u}$ is quasi-additive with respect to $\left\{Q_{j}\right\}$ :

$$
\gamma_{u}(E) \asymp \sum_{j \geq 1} \gamma_{u}\left(E \cap Q_{j}\right), \quad \text { for all Borel } E \subset D .
$$

Proof. (1) Choose $r_{1}$ to be the constant from Definition 5.5 and let $E \subset D \cap B\left(z, r_{1}\right)$. If $E \cap Q_{j} \neq \emptyset$, then $\operatorname{diam}\left(Q_{j}\right)<\operatorname{dist}\left(Q_{j}, \partial D\right)<r_{1}$. By subadditivity of $\gamma_{u}$, we have that $\gamma_{u}(E) \leq \sum_{j} \gamma_{u}\left(E \cap Q_{j}\right)$. For the reverse inequality we may assume that $\gamma_{u}(E)<\infty$. Then there exists a measure $\mu$ such that $k_{u} \mu \geq 1$ on $E$ and 
$\|\mu\| \leq 2 \gamma_{u}(E)$. For each $Q_{j}$ such that $E \cap Q_{j} \neq \emptyset$, we decompose the measure $\mu$ into $\mu_{j}:=\mu_{\mid Q_{j}^{*}}$ and $\mu_{j}^{\prime}:=\mu_{\mid D \backslash Q_{j}^{*}}$. Then either (i) $k_{u} \mu_{j} \geq \frac{1}{2}$ on $E \cap Q_{j}$, or (ii) $k_{u} \mu_{j}^{\prime}(x) \geq \frac{1}{2}$ for some $x \in E \cap Q_{j}$. Let $J_{1}$ denote the set of indices $j$ for which (i) holds, and $J_{2}$ those for which (ii) holds. For $j \in J_{1}$ we have $\gamma_{u}\left(E \cap Q_{j}\right) \leq 2\left\|\mu_{j}\right\|$. Since the number of overlaps of $\left\{Q_{j}^{*}\right\}$ is uniformly bounded, it follows that

$$
\sum_{j \in J_{1}} \gamma_{u}\left(E \cap Q_{j}\right) \leq 2 \sum_{j \in J_{1}}\left\|\mu_{j}\right\| \leq c_{1}\|\mu\| \leq 2 c_{1} \gamma_{u}(E)
$$

For $j \in J_{2}$, by the local Harnack property of $k_{u}$ (Lemma 5.6) we have

$$
\begin{aligned}
k_{u} \mu(y) & \geq k_{u} \mu_{j}^{\prime}(y)=\int_{D \backslash Q_{j}^{*}} k_{u}(y, w) \mu_{j}^{\prime}(d w) \\
& \geq c_{2} \int_{D \backslash Q_{j}^{*}} k_{u}(x, w) \mu_{j}^{\prime}(d w)=c_{2} k_{u} \mu(x) \geq \frac{1}{2} c_{2} .
\end{aligned}
$$

Therefore,

$$
k_{u} \mu \geq \frac{1}{2} c_{2}=: c_{3}^{-1} \quad \text { on } \bigcup_{j \in J_{2}} Q_{j},
$$

implying that $\gamma_{u}\left(\bigcup_{j \in J_{2}} Q_{j}\right) \leq c_{3}\|\mu\| \leq 2 c_{3} \gamma_{u}(E)$. Since by Proposition 5.10 $\sigma_{u}$ is locally comparable to $\gamma_{u}$, it follows from the $\sigma$-additivity of $\sigma_{u}$ that

$$
\begin{aligned}
\sum_{j \in J_{2}} \gamma_{u}\left(E \cap Q_{j}\right) & \leq \sum_{j \in J_{2}} \gamma_{u}\left(Q_{j}\right) \leq c \sum_{j \in J_{2}} \sigma_{u}\left(Q_{j}\right) \\
& \leq c_{4} \sigma_{u}\left(\bigcup_{j \in J_{2}} Q_{j}\right) \leq c_{5} \gamma_{u}\left(\bigcup_{j \in J_{2}} Q_{j}\right) \leq c_{6} \gamma_{u}(E)
\end{aligned}
$$

Together with (5.17) this finishes the proof.

Part (2) is proved analogously.

In the remainder of the section we discuss sufficient geometric conditions which imply the (local) Hardy inequality.

For $v \in \mathcal{F}_{D}$,

$$
\mathcal{E}(v, v)=\int_{D} \int_{D}(v(x)-v(y))^{2} J_{X}(x-y) d y d x+2 \int_{D} v(x)^{2} \kappa_{D}(x) d x,
$$

where $\kappa_{D}$ is given by $\kappa_{D}(x):=\int_{D^{c}} J_{X}(x-y) d y$.

For $x \in D$, let $z_{x}$ be the point on $\partial D$ such that $\left|z_{x}-x\right|=\delta_{D}(x)$. We say $D$ satisfies the local exterior volume condition at $z \in \partial D$ if there exist $r_{0}, c>0$ such that for every $x \in B\left(z, r_{0}\right) \cap D,\left|D^{c} \cap B\left(z_{x}, \delta_{D}(x)\right)\right| \geq c \delta_{D}(x)^{d}$.

Proposition 5.12. The local Hardy inequality holds at $z \in \partial D$ if $D$ is an open set satisfying the local exterior volume condition at $z$.

Proof. Let $x \in B\left(z, r_{0}\right) \cap D$. Then by (1.7),

$$
\kappa_{D}(x) \geq \int_{D^{c} \cap B\left(z_{x}, \delta_{D}(x)\right)}|x-y|^{-d} \Psi\left(|x-y|^{-1}\right) d y .
$$


If $y \in D^{c} \cap B\left(z_{x}, \delta_{D}(x)\right)$, then $|x-y| \leq\left|x-z_{x}\right|+\left|z_{x}-y\right| \leq 2 \delta_{D}(x)$. Hence $\Psi\left(|x-y|^{-1}\right) \geq \Psi\left(2^{-1} \delta_{D}(x)^{-1}\right) \geq c_{3} \Psi\left(\delta_{D}(x)^{-1}\right)$. This implies that

$$
\begin{aligned}
& \int_{D^{c} \cap B\left(z_{x}, \delta_{D}(x)\right)}|x-y|^{-d} \Psi\left(|x-y|^{-1}\right) d y \\
& \geq c_{3} \delta_{D}(x)^{-d} \Psi\left(\delta_{D}(x)^{-1}\right)\left|D^{c} \cap B\left(z_{x}, \delta_{D}(x)\right)\right| \geq c_{4} \Psi\left(\delta_{D}(x)^{-1}\right),
\end{aligned}
$$

where in the last inequality we used the local exterior volume condition at $z$. Thus by (5.18) we have that

$$
\begin{aligned}
\mathcal{E}(v, v) & \geq \int_{D} v(x)^{2} \kappa_{D}(x) d x \geq \int_{B\left(z, r_{0}\right) \cap D} v(x)^{2} \kappa_{D}(x) d x \\
& \geq c_{1} \int_{B\left(z, r_{0}\right) \cap D} v(x)^{2} \Psi\left(\delta_{D}(x)^{-1}\right) d x
\end{aligned}
$$

We say $D$ satisfies the exterior volume condition if there exist $c>0$ such that for every $x \in D,\left|D^{c} \cap B\left(z_{x}, \delta_{D}(x)\right)\right| \geq c \delta_{D}(x)^{d}$, where $z_{x}$ is a point on $\partial D$ such that $\left|z_{x}-x\right|=\delta_{D}(x)$.

Proposition 5.13. Suppose that (H2) holds and that $D$ is either an unbounded $\kappa$-fat open set whose upper Assouad dimension is strictly less than $d-2\left(\delta_{2} \vee \delta_{4}\right)$ (see 17, Definition 2.1] for the definition) or an open set satisfying the exterior volume condition. There exists a constant $c>0$ such that

$$
\mathcal{E}(v, v) \geq c \int_{D} v^{2}(x) \Psi\left(\delta_{D}(x)^{-1}\right) d x, \quad v \in \mathcal{F}_{D} .
$$

Proof. If $D$ is an unbounded $\kappa$-fat open set whose upper Assouad dimension is strictly less than $d-2\left(\delta_{2} \vee \delta_{4}\right)$, then it follows from [17, Theorem 4 and Proposition 9] that

$$
\mathcal{E}(v, v) \geq \int_{D} \int_{D}(v(x)-v(y))^{2} J_{X}(x-y) d y d x \geq c \int_{D} v^{2}(x) \Psi\left(\delta_{D}(x)^{-1}\right) d x .
$$

Since $\kappa_{D}(x) \asymp \int_{D^{c}}|x-y|^{-d} \Psi\left(|x-y|^{-1}\right) d y$, for all $x \in D$, if $D$ is an open set satisfying the exterior volume condition, the proof is similar to that of the previous proposition, so we omit the proof.

\section{Minimal thinness at a Finite Martin Boundary point}

We start this section by recalling the definition of minimal thinness and proving a general result for minimal thinness of a set at any minimal Martin boundary point.

Definition 6.1. Let $D$ be an open set in $\mathbb{R}^{d}$. A set $E \subset D$ is said to be minimally thin in $D$ at $z \in \partial_{m} D$ with respect to $X$ if $\widehat{R}_{M_{D}(\cdot, z)}^{E} \neq M_{D}(\cdot, z)$.

For any $z \in \partial_{M} D$, let $X^{D, z}=\left(X_{t}^{D, z}, \mathbb{P}_{x}^{z}\right)$ denote the $M_{D}(\cdot, z)$-process, Doob's $h$-transform of $X^{D}$ with $h(\cdot)=M_{D}(\cdot, z)$. The lifetime of $X^{D, z}$ will be denoted by $\zeta$. It is known (see 34]) that $\lim _{t \uparrow \zeta} X_{t}^{D, z}=z \mathbb{P}_{x}^{z}$-a.s. For $E \subset D$, let $T_{E}:=$ $\inf \left\{t>0: X_{t}^{D, z} \in E\right\}$. It is proved in [22, Satz 2.6] that a set $E \subset D$ is minimally thin at $z \in \partial_{m} D$ if and only if there exists $x \in D$ such that $\mathbb{P}_{x}^{z}\left(T_{E}<\zeta\right) \neq 1$.

The following proposition gives two more equivalent conditions for minimal thinness. 
Proposition 6.2. Let $D$ be an open set in $\mathbb{R}^{d}, A \subset D$ and $z \in \partial_{m} D$. The following are equivalent:

(1) $A$ is minimally thin in $D$ at $z$ with respect to $X$.

(2) There exists an excessive function $u=G_{D} \mu+M_{D} \nu$ such that

$$
\liminf _{x \rightarrow z, x \in A} \frac{u(x)}{M_{D}(x, z)}>0 .
$$

(3) There exists a potential $u=G_{D} \mu$ such that $\liminf _{x \rightarrow z, x \in A} \frac{u(x)}{M_{D}(x, z)}=+\infty$.

Proof. We sketch the proof following the proof of [4, Theorem 9.2.6]. Clearly, (3) implies (2).

Assume that (2) holds. Then there exists a Martin topology neighborhood $W$ of $z$ and $a>\nu(\{z\})$ such that $u \geq a M_{D}(\cdot, z)$ on $A \cap W$. If $\widehat{R}_{M_{D}(\cdot, z)}^{A \cap W}=M_{D}(\cdot, z)$, then $u \geq \widehat{R}_{u}^{A \cap W} \geq a M_{D}(\cdot, z)$ everywhere. Thus $u-a M_{D}(\cdot, z)$ is excessive; hence $u-a M_{D}(\cdot, z)=G_{D} \mu+M_{D} \widetilde{\nu}$ for a (non-negative) measure $\widetilde{\nu}$ on $\partial D$. On the other hand, $u-a M_{D}(\cdot, z)=G_{D} \mu+M_{D} \nu_{\mid \partial D \backslash\{z\}}+(\nu(\{z\})-a) M_{D}(\cdot, z)$. This implies that $\widetilde{\nu}=\nu_{\mid \partial D \backslash\{z\}}+(\nu(\{z\})-a) \delta_{z}$ yielding $\widetilde{\nu}(\{z\})=\nu(\{z\})-a<0$, which is a contradiction. Hence $\widehat{R}_{M_{D}(\cdot, z)}^{A \cap W} \neq M_{D}(\cdot, z)$; i.e., $A$ is minimally thin at $z$. Thus (2) implies (1).

Suppose that (1) holds. By [22, Lemma 2.7], there exists an open subset $U \subset \mathbb{R}^{d}$ such that $A \subset U$, and $U$ is minimally thin in $D$ at $z$ with respect to $X$. By the analog of [4, Theorem 9.2.5], there is a decreasing sequence $\left(W_{n}\right)_{n \geq 1}$ of Martin topology open neighborhoods of $z$ shrinking to $z$ and such that $\widehat{R}_{M_{D}(\cdot, z)}^{U \cap W_{n}}\left(x_{0}\right) \leq 2^{-n}$. Let $u_{1}:=\sum_{n=1}^{\infty} \widehat{R}_{M_{D}(\cdot, z)}^{U \cap W_{n}}$. Then $u_{1}$ is a sum of potentials, hence a potential itself since $u_{1}\left(x_{0}\right)<\infty$. Further, $\widehat{R}_{M_{D}(\cdot, z)}^{U \cap W_{n}}=M_{D}(\cdot, z)$ on the open set $U \cap W_{n}$. Therefore, $u_{1}(x) / M_{D}(x, z) \rightarrow \infty$ as $x \rightarrow z, x \in U$. Thus (3) holds.

Note that this proposition holds true regardless of whether $z$ is a finite or an infinite Martin boundary point.

In the sequel we assume that $D$ is a $\kappa$-fat open set with localization radius $R$ and that $z$ is a fixed point in $\partial D$. Without loss of generality we assume $R<1 / 10$. Recall that $x_{0} \in D \cap B(z, R)$ satisfies $\kappa R<\delta_{D}\left(x_{0}\right)<R$. Let $M_{D}$ be the Martin kernel of $D$ based at $x_{0}$.

Lemma 6.3. There exists $C_{6}=C_{6}(\Psi, \gamma, R, \kappa)>0$ such that for every $x, y \in$ $B\left(z, 2^{-7} \kappa^{2} R\right) \cap D$ with $|x-y| \geq \frac{3}{4}|x-z|$,

$$
\frac{G_{D}(x, y)}{M_{D}(x, z)} \leq C_{6} G_{D}\left(x_{0}, y\right) .
$$

Proof. Recall that $C_{3}$ and $C_{4}$ are the constants from Theorem 2.11 and Theorem 3.2 respectively, and that $g(\cdot)=G_{D}\left(\cdot, x_{0}\right)$ on $B(z, \kappa R / 4)$. We have

$$
\frac{G_{D}(x, y)}{M_{D}(x, z)} \leq C_{3} C_{4} \frac{\Phi(|x-y|)|x-z|^{d}}{\Phi(|x-z|)|x-y|^{d}} \frac{G_{D}\left(y, x_{0}\right) G_{D}\left(A_{|x-z|}(z), x_{0}\right)^{2}}{G_{D}\left(A, x_{0}\right)^{2}},
$$

where $A \in \mathcal{B}(x, y)$ and $\mathcal{B}(x, y)$ is defined by (2.5). Since $|x-y| \geq \frac{3}{4}|x-z|$, we have $r(x, y)=\delta_{D}(x) \vee \delta_{D}(y) \vee|x-y| \geq \frac{3}{4}|x-z|$. 
By the definition of $A_{\frac{4}{3} r(x, y)}(z), \delta_{D}\left(A_{\frac{4}{3} r(x, y)}(z)\right) \geq \kappa \frac{4}{3} r(x, y)>\kappa r(x, y) / 2$. Moreover,

$$
\left|x-A_{\frac{4}{3} r(x, y)}(z)\right| \leq|x-z|+\left|z-A_{\frac{4}{3} r(x, y)}(z)\right| \leq \frac{4}{3}|x-y|+\frac{4}{3} r(x, y)<3 r(x, y)
$$

and $\left|y-A_{\frac{4}{3} r(x, y)}(z)\right| \leq|y-x|+\left|x-A_{\frac{4}{3} r(x, y)}(z)\right|<4 r(x, y)<5 r(x, y)$. Hence $A_{\frac{4}{3} r(x, y)}(z) \in \mathcal{B}(x, y)$.

By Lemma 2.7 and Lemma 2.9(2),

$G_{D}\left(A_{|x-z|}(z), x_{0}\right) \leq c_{1} G_{D}\left(A_{\frac{4}{3} r(x, y)}(z), x_{0}\right)=c_{2} G_{D}\left(A_{\frac{4}{3} r(x, y)}(z), x_{0}\right) \leq c_{3} G_{D}\left(A, x_{0}\right)$.

Moreover, by (2.2),$\Phi(|x-y|)|x-z|^{d} /\left(\Phi(|x-z|)|x-y|^{d}\right) \leq c_{4}$. The assertion of the lemma now follows from this, (6.2) and (6.3).

The following proposition is an analog of [6, Proposition V, 4.15]. For $E \subset D$, define $E_{n}=E \cap\left\{x \in D: 2^{-n-1} \leq|x-z|<2^{-n}\right\}, n \geq 1$.

Proposition 6.4. A set $E \subset D$ is minimally thin in $D$ at $z$ with respect to $X$ if and only if

$$
\sum_{n=1}^{\infty} R_{M_{D}(\cdot, z)}^{E_{n}}\left(x_{0}\right)<\infty
$$

Proof. Assume $\sum_{n=1}^{\infty} R_{M_{D}(\cdot, z)}^{E_{n}}\left(x_{0}\right)<\infty$. Then there exists $n_{0} \in \mathbb{N}$ such that

$$
\sum_{n_{0}}^{\infty} R_{M_{D}(\cdot, z)}^{E_{n}}\left(x_{0}\right)<\frac{1}{2} M_{D}\left(x_{0}, z\right) .
$$

Let $B=B\left(z, 2^{-n_{0}}\right)$. Then $A:=B \cap E \subset \bigcup_{n_{0}}^{\infty} E_{n}$. Therefore,

$$
R_{M_{D}(\cdot, z)}^{A}\left(x_{0}\right) \leq \sum_{n_{0}}^{\infty} R_{M_{D}(\cdot, z)}^{E_{n}}\left(x_{0}\right)<\frac{1}{2} M_{D}\left(x_{0}, z\right),
$$

implying $\widehat{R}_{M_{D}(\cdot, z)}^{A}\left(x_{0}\right)<\frac{1}{2} M_{D}\left(x_{0}, z\right)$. Hence, $A$ is minimally thin in $D$ at $z$ with respect to $X$. Clearly, $E$ is also minimally thin in $D$ at $z$ with respect to $X$.

Conversely, suppose that $E$ is minimally thin in $D$ at $z$ with respect to $X$. By Proposition 6.2, there exists a potential $u$ such that $\liminf _{x \rightarrow z, x \in E} \frac{u(x)}{M_{D}(x, z)}=+\infty$. Without loss of generality, we may assume that $u\left(x_{0}\right) \leq\left(2 C_{6}\right)^{-1}$. There exists $n_{1} \in \mathbb{N}$ with $2^{-n_{1}} \leq 2^{-7} \kappa^{2} R$ such that $u(x)>M_{D}(x, z)$ for all $x \in E \cap B\left(z, 2^{-n_{1}}\right)$. Thus, $E \subset \bar{B}\left(z, 2^{-n_{1}}\right)^{c} \cup\left\{u>M_{D}(\cdot, z)\right\}$. For $n \geq n_{1}$ define

$$
F_{n}=\left\{x \in D: 2^{-n-1}<|x-z|<2^{-n}, u(x)>M_{D}(x, z)\right\} \quad \text { and } \quad F=\bigcup_{n_{1}}^{\infty} F_{n} .
$$

Let $x \in E_{n}$. Since $|x-z| \leq 2^{-n_{1}}$, we have that $u(x)>M_{D}(x, z)$ and thus $x \in F_{n}$. This shows that $E_{n} \subset F_{n}, n \geq n_{1}$. Therefore, it suffices to show that $\sum_{n_{1}}^{\infty} R_{M_{D}(\cdot, z)}^{F_{n}}\left(x_{0}\right)<\infty$. Since $u>M_{D}(\cdot, z)$ on $F$, it follows that $R_{M_{D}(\cdot, z)}^{F}\left(x_{0}\right) \leq$ $u\left(x_{0}\right)$.

Let $i \in\{1,2,3\}$. For every $n \in \mathbb{N}$, let $U_{n}=F_{n_{1}+3 n+i}$. Since $i \in\{1,2,3\}$ is arbitrary, it suffices to show that $\sum_{n=1}^{\infty} R_{M_{D}(\cdot, z)}^{U_{n}}\left(x_{0}\right)<\infty$. Let $U=\bigcup_{n=1}^{\infty} U_{n}$. 
Then $U \subset F$ and thus $R_{M_{D}(\cdot, z)}^{U}\left(x_{0}\right) \leq u\left(x_{0}\right)$. Note that since $U$ is open, $\widehat{R}_{M_{D}(\cdot, z)}^{U}=$ $R_{M_{D}(\cdot, z)}^{U}$ (see [6, p. 205]). Since $u$ is a potential, the same holds for $\widehat{R}_{M_{D}(\cdot, z)}^{U}$; hence there exists a measure $\mu$ such that $R_{M_{D}(\cdot, z)}^{U}=G_{D} \mu$. Moreover, since $R_{M_{D}(\cdot, z)}^{U}$ is harmonic on $\bar{U}^{c}$ (cf. [6, III.2.5]), $\mu\left(U^{c}\right)=0$. Let $\mu_{n}:=\mu_{\mid \bar{U}_{n}}$. Since $\bar{U}_{n}$ are pairwise disjoint,

$$
\mu=\sum_{n=1}^{\infty} \mu_{n} \quad \text { and } \quad G_{D} \mu=\sum_{n=1}^{\infty} G_{D} \mu_{n} .
$$

Fix $n \in \mathbb{N}$ and consider $l \in \mathbb{N}, x \in U_{n}, y \in \bar{U}_{l}$. If $l>n$, then

$$
|y-z|<2^{-n_{1}-3 l-i} \leq 2^{1-3(l-n)}|x-z| \leq \frac{1}{4}|x-z|,
$$

and hence $|x-y| \geq|x-z|-|y-z| \geq \frac{3}{4}|x-z|$. If $l<n$, then analogously, $|x-z| \leq \frac{1}{4}|y-z|$, hence $|x-y| \geq|y-z|-|x-z| \geq \frac{3}{4}|y-z| \geq \frac{3}{4}|x-z|$. Thus, in both cases,

$$
|x-y| \geq \frac{3}{4}|x-z| \geq \frac{3}{4} \delta_{D}(x), \quad x \in U_{n}, y \in \overline{U_{l}}, l \neq n .
$$

Define $\mu_{n}^{\prime}=\mu-\mu_{n}$ and let $x \in U_{n}$. We have

$$
G_{D} \mu_{n}^{\prime}(x)=\int_{D} G_{D}(x, y) \mu_{n}^{\prime}(d y)=M_{D}(x, z) \int_{D} \frac{G_{D}(x, y)}{M_{D}(x, z)} \mu_{n}^{\prime}(d y)
$$

By (6.1) we have that

$$
\begin{aligned}
& G_{D} \mu_{n}^{\prime}(x)=M_{D}(x, z) \int_{D} \frac{G_{D}(x, y)}{M_{D}(x, z)} \mu_{n}^{\prime}(d y) \leq C_{6} M_{D}(x, z) \int_{D} G_{D}\left(x_{0}, y\right) \mu_{n}^{\prime}(d y) \\
& \leq C_{6} M_{D}(x, z) G_{D} \mu\left(x_{0}\right) \leq C_{6} M_{D}(x, z) u\left(x_{0}\right)<\frac{1}{2} M_{D}(x, z) .
\end{aligned}
$$

Since $G_{D} \mu_{n}+G_{D} \mu_{n}^{\prime}=G_{D} \mu=R_{M_{D}(\cdot, z)}^{U}=M_{D}(\cdot, z)$ on $U$, it follows that $G_{D} \mu_{n}=$ $M_{D}(\cdot, z)-G_{D} \mu_{n}^{\prime} \geq M_{D}(\cdot, z)-\frac{1}{2} M_{D}(\cdot, z)=\frac{1}{2} M_{D}(\cdot, z)$ on $U_{n}$. This implies that $G_{D} \mu_{n} \geq \frac{1}{2} R_{M_{D}(\cdot, z)}^{U_{n}}$. Finally,

$$
\sum_{n=1}^{\infty} R_{M_{D}(\cdot, z)}^{U_{n}}\left(x_{0}\right) \leq 2 \sum_{n=1}^{\infty} G_{D} \mu_{n}\left(x_{0}\right)=2 G_{D} \mu\left(x_{0}\right)<\infty .
$$

By Theorem 3.2, for large $n$,

$$
C_{4}^{-1} 2^{n d} \frac{g(x) \Phi\left(2^{-n}\right)}{g\left(A_{2^{-n}}(z)\right)^{2}} \leq M_{D}(x, z) \leq C_{4} 2^{(n+1) d} \frac{g(x) \Phi\left(2^{-n}\right)}{g\left(A_{2^{-n}}(z)\right)^{2}}, \quad x \in E_{n} .
$$

This implies that

$$
C_{4}^{-1} 2^{n d} \frac{\Phi\left(2^{-n}\right)}{g\left(A_{2^{-n}}(z)\right)^{2}} R_{g}^{E_{n}} \leq R_{M_{D}(\cdot, z)}^{E_{n}} \leq C_{4} 2^{(n+1) d} \frac{\Phi\left(2^{-n}\right)}{g\left(A_{2^{-n}}(z)\right)^{2}} R_{g}^{E_{n}} .
$$

In particular,

$$
\sum_{n=1}^{\infty} R_{M_{D}(\cdot, z)}^{E_{n}}\left(x_{0}\right)<\infty \quad \text { if and only if } \quad \sum_{n=1}^{\infty} 2^{n d} \frac{\Phi\left(2^{-n}\right)}{g\left(A_{2^{-n}}(z)\right)^{2}} R_{g}^{E_{n}}\left(x_{0}\right)<\infty
$$


Note that $\widehat{R}_{g}^{E_{n}}$ is a potential; hence there exists a measure $\lambda_{n}$ (supported by $\bar{E}_{n}$ ) such that $\widehat{R}_{g}^{E_{n}}=G_{D} \lambda_{n}$. Also, $\widehat{R}_{g}^{E_{n}}=g=G_{D}\left(\cdot, x_{0}\right)$ on $E_{n}$ (except for a polar set, and at least for large $n$ ), hence

$$
\begin{aligned}
\widehat{R}_{g}^{E_{n}}\left(x_{0}\right) & =G_{D} \lambda_{n}\left(x_{0}\right)=\int_{\bar{E}_{n}} G_{D}\left(x_{0}, y\right) \lambda_{n}(d y)=\int_{\bar{E}_{n}} g(y) \lambda_{n}(d y) \\
& =\int_{\bar{E}_{n}} \widehat{R}_{g}^{E_{n}}(y) \lambda_{n}(d y)=\int_{D} \int_{D} G_{D}(x, y) \lambda_{n}(d y) \lambda_{n}(d x)=\gamma_{g}\left(E_{n}\right) .
\end{aligned}
$$

We conclude from (6.5) that

$$
\sum_{n=1}^{\infty} R_{M_{D}(\cdot, z)}^{E_{n}}\left(x_{0}\right)<\infty \quad \text { if and only if } \sum_{n=1}^{\infty} 2^{n d} \frac{\Phi\left(2^{-n}\right)}{g\left(A_{2^{-n}}(z)\right)^{2}} \gamma_{g}\left(E_{n}\right)<\infty .
$$

Thus we have proved the following Wiener-type criterion for minimal thinness.

Corollary 6.5. $E \subset D$ is minimally thin in $D$ at $z$ with respect to $X$ if and only if

$$
\sum_{n=1}^{\infty} 2^{n d} \frac{\Phi\left(2^{-n}\right)}{g\left(A_{2^{-n}}(z)\right)^{2}} \gamma_{g}\left(E_{n}\right)<\infty
$$

Now we prove a version of Aikawa's criterion for minimal thinness.

Proposition 6.6. Let $E \subset D$. Let $\left\{Q_{j}\right\}$ be a Whitney decomposition of $D$ and let $x_{j}$ denote the center of $Q_{j}$. If $\left(\mathcal{E}, \mathcal{F}_{D}\right)$ satisfies the local Hardy inequality with a localization constant $r_{0}$ at $z \in \partial D$, then $E$ is minimally thin in $D$ at $z$ with respect to $X$ if and only if

$$
\sum_{j: Q_{j} \cap B\left(z, r_{0} / 2\right) \neq \emptyset} \operatorname{dist}\left(z, Q_{j}\right)^{-d} \frac{\Phi\left(\operatorname{dist}\left(z, Q_{j}\right)\right)}{g\left(A_{\operatorname{dist}\left(z, Q_{j}\right)}(z)\right)^{2}} g\left(x_{j}\right)^{2} \operatorname{Cap}_{D}\left(E \cap Q_{j}\right)<\infty .
$$

Proof. Let $r_{1}:=r_{0} / 2$. Further, let $V_{n}=\left\{x \in \mathbb{R}^{d}: 2^{-n-1} \leq|x-z|<2^{-n}\right\}$ so that $E_{n}=E \cap V_{n}$. If $V_{n} \cap Q_{j} \neq \emptyset$, then $\operatorname{dist}\left(z, Q_{j}\right) \asymp 2^{-n}$. Since $g$ satisfies the local scale invariant Harnack inequality, by the local quasi-additivity of $\gamma_{g}$ at $z$ (Proposition $5.11(1))$,

$$
\begin{aligned}
& \sum_{n=1}^{\infty} 2^{n d} \frac{\Phi\left(2^{-n}\right)}{g\left(A_{2^{-n}}(z)\right)^{2}} \gamma_{g}\left(E_{n}\right) \asymp \sum_{n=1}^{\infty} 2^{n d} \frac{\Phi\left(2^{-n}\right)}{g\left(A_{2^{-n}}(z)\right)^{2}} \sum_{Q_{j} \cap B\left(z, r_{1}\right) \neq \emptyset} \gamma_{g}\left(E_{n} \cap Q_{j}\right) \\
\asymp & \sum_{j: Q_{j} \cap B\left(z, r_{1}\right) \neq \emptyset} \sum_{n: V_{n} \cap Q_{j} \neq \emptyset} \operatorname{dist}\left(z, Q_{j}\right)^{-d} \frac{\Phi\left(\operatorname{dist}\left(z, Q_{j}\right)\right)}{g\left(A_{\operatorname{dist}\left(z, Q_{j}\right)}(z)\right)^{2}} \gamma_{g}\left(E_{n} \cap Q_{j}\right) \\
= & \sum_{Q_{j} \cap B\left(z, r_{1}\right) \neq \emptyset} \operatorname{dist}\left(z, Q_{j}\right)^{-d} \frac{\Phi\left(\operatorname{dist}\left(z, Q_{j}\right)\right)}{g\left(A_{\operatorname{dist}\left(z, Q_{j}\right)}(z)\right)^{2}} \sum_{n: V_{n} \cap Q_{j} \neq \emptyset} \gamma_{g}\left(E_{n} \cap Q_{j}\right) \\
\asymp & \sum_{Q_{j} \cap B\left(z, r_{1}\right) \neq \emptyset} \operatorname{dist}\left(z, Q_{j}\right)^{-d} \frac{\Phi\left(\operatorname{dist}\left(z, Q_{j}\right)\right)}{g\left(A_{\operatorname{dist}\left(z, Q_{j}\right)}(z)\right)^{2}} \gamma_{g}\left(E \cap Q_{j}\right) .
\end{aligned}
$$

In the second line above we used the fact that $g\left(A_{2^{-n}}(z)\right)$ and $g\left(A_{\operatorname{dist}\left(z, Q_{j}\right)}(z)\right)$ are comparable, which is a consequence of 33 , Theorem 2.2]. For the last line we argue as follows: One inequality is the subadditivity of capacity. For the other note that there exists $N \in \mathbb{N}$ such that for every $Q_{j}, \sum_{n, V_{n} \cap Q_{j} \neq \emptyset} 1=\sum_{n} 1_{V_{n} \cap Q_{j}} \leq N$. Hence, $\sum_{n, V_{n} \cap Q_{j} \neq \emptyset} \gamma_{g}\left(E \cap V_{n} \cap Q_{j}\right) \leq \sum_{n, V_{n} \cap Q_{j} \neq \emptyset} \gamma_{g}\left(E \cap Q_{j}\right) \leq N \gamma_{g}\left(E \cap Q_{j}\right)$. 
Finally, by Lemma 5.8 we see that $\gamma_{g}\left(E \cap Q_{j}\right) \asymp g\left(x_{j}\right)^{2} \operatorname{Cap}_{D}\left(E \cap Q_{j}\right)$, which completes the proof by Corollary 6.5 .

The next result is an analog of [2, Part II, Corollary 7.4.4].

Corollary 6.7. Suppose that either (i) $D$ is a half-space, or (ii) $D \subset \mathbb{R}^{d}$ is a $C^{1,1}$ open set and $\gamma=1$ in (1.2). Let $x_{j}$ denote the center of $Q_{j}$. Then $E$ is minimally thin in $D$ at $z \in \partial D$ with respect to $X$ if and only if

$$
\sum_{j: Q_{j} \cap B(z, 1) \neq \emptyset} \operatorname{dist}\left(z, Q_{j}\right)^{-d} \Phi\left(\operatorname{dist}\left(Q_{j}, \partial D\right)\right) \operatorname{Cap}_{D}\left(E \cap Q_{j}\right)<\infty .
$$

Proof. The function $g$ is harmonic in $D \cap B\left(z, 2 r_{1}\right)$ where $r_{1}:=\kappa R / 4$. Since $X$ satisfies (H1), applying [33, Theorem 2.3(i)], we get that for $Q_{j} \cap B\left(z, r_{1} / 10\right) \neq \emptyset$,

$$
g\left(A_{\operatorname{dist}\left(z, Q_{j}\right)}(z)\right) \asymp \mathbb{E}_{A_{\mathrm{dist}\left(z, Q_{j}\right)}(z)}\left[\tau_{D \cap B\left(z, 2 r_{1}\right)}\right] \int_{B\left(z, r_{1}\right)^{c}} j(|y-z|) g(y) d y
$$

and

$$
g\left(x_{j}\right) \asymp \mathbb{E}_{x_{j}}\left[\tau_{D \cap B\left(z, 2 r_{1}\right)}\right] \int_{B\left(z, r_{1}\right)^{c}} j(|y-z|) g(y) d y .
$$

Suppose that $D$ is a $C^{1,1}$ open set and $\gamma=1$. Since a $C^{1,1}$ set satisfies the interior and exterior ball condition, by combining (6.7) and (6.8) with [9], Theorem 4.1 and Corollary 4.5], we get

$$
g\left(A_{\operatorname{dist}\left(z, Q_{j}\right)}(z)\right) \asymp \Phi\left(\operatorname{dist}\left(z, Q_{j}\right)\right)^{1 / 2} \quad \text { and } \quad g\left(x_{j}\right) \asymp \Phi\left(\operatorname{dist}\left(Q_{j}, \partial D\right)\right)^{1 / 2} .
$$

In the case when $D$ is the half-space $\mathbb{H}$, the two relations above are an immediate consequence of [9, Proposition 2.4], the boundary Harnack principle in [33, Theorem 2.3(ii)] and the fact that $V(x):=V\left(x_{d}\right)$, where $V$ is the renewal function of the ascending ladder height process of the $d$-th component of $X$, is harmonic in $\mathbb{H}$ with respect to $X$. Thus the corollary follows immediately from Proposition 6.6.

Proof of Theorem 1.3. Let $r_{1}:=r_{0} / 2$ and without loss of generality assume $r_{0}<$ $\kappa R / 4$. Assume that $E$ is minimally thin in $D$ at $z$ with respect to $X$. Recall that $g(x):=G_{D}\left(x, x_{0}\right) \wedge C_{2}$ and $g(\cdot)=G_{D}\left(\cdot, x_{0}\right)$ on $B(z, \kappa R / 4)$. Thus, by Proposition 6.6 ,

$\sum_{j: Q_{j} \cap B\left(z, r_{1}\right) \neq \emptyset} \operatorname{dist}\left(z, Q_{j}\right)^{-d} \frac{\Phi\left(\operatorname{dist}\left(z, Q_{j}\right)\right)}{G_{D}\left(A_{\operatorname{dist}\left(z, Q_{j}\right)}(z), x_{0}\right)^{2}} G_{D}\left(x_{j}, x_{0}\right)^{2} \operatorname{Cap}_{D}\left(E \cap Q_{j}\right)<\infty$.

First note that for $Q_{j} \cap B\left(z, r_{1}\right) \neq \emptyset$,

$$
\operatorname{Cap}_{D}\left(E \cap Q_{j}\right) \geq c_{1} \sigma\left(E \cap Q_{j}\right)=c_{1} \int_{E} \mathbf{1}_{Q_{j}}(x) \Psi\left(\delta_{D}(x)^{-1}\right) d x
$$


Next, $\operatorname{dist}\left(z, Q_{j}\right) \asymp|x-z|$ and $G_{D}\left(x_{j}, x_{0}\right) \asymp G_{D}\left(x, x_{0}\right)$ for $x \in Q_{j}$. Therefore,

$$
\begin{aligned}
& \sum_{Q_{j} \cap B\left(z, r_{1}\right) \neq \emptyset} \operatorname{dist}\left(z, Q_{j}\right)^{-d} \frac{\Phi\left(\operatorname{dist}\left(z, Q_{j}\right)\right)}{G_{D}\left(A_{\operatorname{dist}\left(z, Q_{j}\right)}(z), x_{0}\right)^{2}} G_{D}\left(x_{j}, x_{0}\right)^{2} \operatorname{Cap}_{D}\left(E \cap Q_{j}\right) \\
\geq & c_{2} \sum_{Q_{j} \cap B\left(z, r_{1}\right) \neq \emptyset} \int_{E}|x-z|^{-d} \frac{\Phi(|x-z|)}{G_{D}\left(A_{|x-z|}(z), x_{0}\right)^{2}} G_{D}\left(x, x_{0}\right)^{2} \mathbf{1}_{Q_{j}}(x) \Psi\left(\delta_{D}(x)^{-1}\right) d x \\
\asymp & \sum_{Q_{j} \cap B\left(z, r_{1}\right) \neq \emptyset} \int_{E}|x-z|^{-d}\left(\frac{G_{D}\left(x, x_{0}\right)}{G_{D}\left(A_{|x-z|}(z), x_{0}\right)}\right)^{2} \frac{\Psi\left(\delta_{D}(x)^{-1}\right)}{\Psi\left(|x-z|^{-1}\right)} \mathbf{1}_{Q_{j}}(x) d x \\
= & \int_{E} \sum_{Q_{j} \cap B\left(z, r_{1}\right) \neq \emptyset}|x-z|^{-d}\left(\frac{G_{D}\left(x, x_{0}\right)}{G_{D}\left(A_{|x-z|}(z), x_{0}\right)}\right)^{2} \frac{\Psi\left(\delta_{D}(x)^{-1}\right)}{\Psi\left(|x-z|^{-1}\right)} \mathbf{1}_{Q_{j}}(x) d x \\
\geq & \int_{E \cap B\left(z, r_{1}\right)}\left(\frac{G_{D}\left(x, x_{0}\right)}{G_{D}\left(A_{|x-z|}(z), x_{0}\right)}\right)^{2} \frac{\Psi\left(\delta_{D}(x)^{-1}\right)}{\Psi\left(|x-z|^{-1}\right)}|x-z|^{-d} d x .
\end{aligned}
$$

Conversely, assume that $E$ is the union of a subfamily of Whitney cubes. Then $E \cap$ $Q_{j}$ is either empty or equal to $Q_{j}$. Since $\operatorname{Cap}_{D}\left(Q_{j}\right) \asymp \sigma\left(Q_{j}\right)=\int_{Q_{j}} \Psi\left(\delta_{D}(x)^{-1}\right) d x$ for $Q_{j} \cap B\left(z, r_{1}\right) \neq \emptyset$ by Proposition 5.11 (1), we can reverse the first inequality in the display above to conclude that

$$
\begin{aligned}
& \sum_{j: Q_{j} \cap B\left(z, r_{1}\right) \neq \emptyset} \operatorname{dist}\left(z, Q_{j}\right)^{-d} \frac{\Phi\left(\operatorname{dist}\left(z, Q_{j}\right)\right)}{G_{D}\left(A_{\operatorname{dist}\left(z, Q_{j}\right)}(Q), x_{0}\right)^{2}} G_{D}\left(x_{j}, x_{0}\right)^{2} \operatorname{Cap}_{D}\left(E \cap Q_{j}\right) \\
& \leq c_{3} \int_{E \cap B\left(z, 5 r_{1}\right)}\left(\frac{G_{D}\left(x, x_{0}\right)}{G_{D}\left(A_{|x-z|}(z), x_{0}\right)}\right)^{2} \frac{\Psi\left(\delta_{D}(x)^{-1}\right)}{\Psi\left(|x-z|^{-1}\right)}|x-z|^{-d} d x
\end{aligned}
$$

Proof of Corollary 1.5. We have seen from the proof of Corollary 6.7 that there exists $r>0$ such that for $Q_{j} \cap B(z, r) \neq \emptyset$,

$$
g\left(A_{\operatorname{dist}\left(z, Q_{j}\right)}(z)\right)=G_{D}\left(A_{\operatorname{dist}\left(z, Q_{j}\right)}(z), x_{0}\right) \asymp \Phi\left(\operatorname{dist}\left(z, Q_{j}\right)\right)^{1 / 2}
$$

and

$$
g\left(x_{j}\right)=G_{D}\left(x_{j}, x_{0}\right) \asymp \Phi\left(\operatorname{dist}\left(Q_{j}, \partial D\right)\right)^{1 / 2} .
$$

Combining the two relations above with the proof of Theorem 1.3 , we immediately arrive at the conclusion of Corollary 1.5

\section{Minimal thinness at Infinity}

Throughout this section we assume that (H2) holds and the constant $\gamma$ in (1.2) is 1. Thus $X$ is a unimodal Lévy process satisfying the global weak scaling conditions in [9, 10. We will establish results for minimal thinness at infinity. Even though the results are analogous to those of the previous section, their proofs contain nontrivial modifications. In particular we will use the recently established boundary Harnack principles given in Theorems 4.3 and 4.4. Thus we include all details except in the proof of Theorem 7.1

We first extend the main result in 32 . Let $\kappa \in(0,1 / 2]$. We say that an open set $D$ in $\mathbb{R}^{d}$ is $\kappa$-fat at infinity if there exists $R>0$ such that for every $r \in[R, \infty)$ there exists $A_{r} \in \mathbb{R}^{d}$ such that $B\left(A_{r}, \kappa r\right) \subset D \cap \bar{B}(0, r)^{c}$ and $\left|A_{r}\right|<\kappa^{-1} r$. 
Theorem 7.1. The Martin boundary at infinity with respect to $X$ of any open set $D$ which is $\kappa$-fat at infinity consists of exactly one point $\infty$. The point $\infty$ is a minimal Martin boundary point.

Proof. The theorem is proved in 32 when $X$ is a subordinate Brownian motion with Lévy exponent $\Psi(\xi)=\phi\left(|\xi|^{2}\right)$ where $\phi$ is a complete Bernstein function satisfying (H1) and (H2). The method in $[32$ is quite robust and can be applied to unimodal Lévy processes satisfying the global weak scaling conditions. In fact, since we have (4.1), 33. Lemma 2.2], (4.2) and (4.3) (instead of 32, (2.2), Lemma 2.2, (2.8) and (2.9)] respectively), using Theorems 4.3 and 4.4 instead of [30, Theorem 1.1] and [31, Theorem], one can follow the proofs in [32, Section 3] line by line and obtain the corresponding results in [32, Section 3]. Once we get the corresponding results in [32, Section 3], then all arguments and results in [32, Section 4] stay the same so that the theorem holds. We omit the details since these would be a simple repetition of proofs in 32 .

Since half-space-like open sets are $\kappa$-fat at infinity, the Martin boundary at infinity with respect to $X$ of any half-space-like open set consists of exactly one point $\infty$, and this point is a minimal Martin boundary point.

In the remainder of this section we assume that $D \subset \mathbb{R}^{d}$ is a half-space-like open set and that $\mathbb{H}_{1} \subset D \subset \mathbb{H}$. Let $x_{0}=(\widetilde{0}, 5)$ and let $M_{D}$ be the Martin kernel of $D$ based at $x_{0}$.

Before we prove Proposition 7.3, which is an analog of Proposition 6.4 at infinity, we establish an inequality involving Green functions and the Martin kernel at infinity. We recall from [10,12] that for the half-space $\mathbb{H}$ we have the following estimates: There exists a constant $c \geq 1$ such that

$$
G_{\mathbb{H}}(x, y) \asymp \frac{\Phi(|x-y|)}{|x-y|^{d}}\left(1 \wedge \frac{\Phi\left(\delta_{\mathbb{H}}(x)\right)}{\Phi(|x-y|)}\right)^{1 / 2}\left(1 \wedge \frac{\Phi\left(\delta_{\mathbb{H}}(y)\right)}{\Phi(|x-y|)}\right)^{1 / 2} .
$$

This implies

$$
c^{-1} \Phi\left(\delta_{\mathbb{H}}(x)\right)^{1 / 2} \leq M_{\mathbb{H}}(x, \infty) \leq c \Phi\left(\delta_{\mathbb{H}}(x)\right)^{1 / 2} .
$$

Relation (7.1) also implies that for every $u, v \in \mathbb{H}_{2}$,

$$
G_{\mathbb{H}_{1}}(u, v) \geq c_{1} \frac{\Phi(|u-v|)}{|u-v|^{d}}\left(1 \wedge \frac{\Phi\left(\delta_{\mathbb{H}}(u)\right)}{\Phi(|u-v|)}\right)^{1 / 2}\left(1 \wedge \frac{\Phi\left(\delta_{\mathbb{H}}(v)\right)}{\Phi(|u-v|)}\right)^{1 / 2} \geq c_{2} G_{\mathbb{H}}(u, v) .
$$

Moreover, if $|x| \geq 10$, then $\left|x-x_{0}\right| \asymp|x| \geq \delta_{\mathbb{H}}(x)$. Thus for $|x| \geq 10$,

$$
G_{\mathbb{H}}\left(x, x_{0}\right) \asymp \frac{\Phi(|x|)}{|x|^{d}}\left(1 \wedge \frac{\Phi\left(\delta_{\mathbb{H}}(x)\right)}{\Phi(|x|)}\right)^{1 / 2}\left(1 \wedge \frac{1}{\Phi(|x|)}\right)^{1 / 2} \asymp \frac{\Phi\left(\delta_{\mathbb{H}}(x)\right)^{1 / 2}}{|x|^{d}} .
$$

Lemma 7.2. There exists $C_{7}>0$ such that for every $x, y \in B(0,10)^{c} \cap D$ with $|x-y| \geq \frac{3}{4}|y|$,

$$
G_{D}(x, y) \leq C_{7} G_{D}\left(x_{0}, y\right) M_{D}(x, \infty) .
$$

Proof. We claim that for every $w \in B(0,10(|x| \vee|y|))^{c} \cap D$ with $\delta_{D}(w) \geq 3$,

$$
\frac{G_{D}(x, y)}{G_{D}(x, w)} \leq c_{1} \frac{G_{D}\left(x_{0}, y\right)}{G_{D}\left(x_{0}, w\right)} .
$$

By letting $w \rightarrow \infty$ with $\delta_{D}(w) \geq 3$, this implies (17.5) immediately.

We prove (7.6) through 3 steps. 
Step 1. We first prove (7.6) for $\mathbb{H}$. Since $|x-w| \asymp\left|x_{0}-w\right|$ and $\delta_{\mathbb{H}}(x) \leq|x| \leq$ $|w|-|x| \leq|x-w|$, by (7.1),

$$
\begin{aligned}
\frac{G_{\mathbb{H}}(x, y) G_{\mathbb{H}}\left(x_{0}, w\right)}{G_{\mathbb{H}}(x, w)} \leq & c_{2} \frac{\Phi(|x-y|)}{|x-y|^{d}}\left(\frac{\Phi\left(\delta_{\mathbb{H}}(x)\right)}{\Phi(|x-y|)}\right)^{1 / 2}\left(\frac{\Phi\left(\delta_{\mathbb{H}}(y)\right)}{\Phi(|x-y|)}\right)^{1 / 2} \\
& \times\left(\frac{\Phi\left(\delta_{\mathbb{H}}\left(x_{0}\right)\right)}{\Phi\left(\left|x_{0}-w\right|\right)}\right)^{1 / 2}\left(\frac{\Phi\left(\delta_{\mathbb{H}}(x)\right)}{\Phi(|x-w|)}\right)^{-1 / 2} \\
\leq & c_{3} \frac{\Phi\left(\delta_{\mathbb{H}}(x)\right)^{1 / 2} \Phi\left(\delta_{\mathbb{H}}(y)\right)^{1 / 2}}{|x-y|^{d} \Phi\left(\delta_{\mathbb{H}}(x)\right)^{1 / 2}}=c_{3} \frac{\Phi\left(\delta_{\mathbb{H}}(y)\right)^{1 / 2}}{|x-y|^{d}} .
\end{aligned}
$$

Thus, by our assumption $|x-y| \geq \frac{3}{4}|y|$ and (7.4),

$$
\frac{G_{\mathbb{H}}(x, y) G_{\mathbb{H}}\left(x_{0}, w\right)}{G_{\mathbb{H}}(x, w)} \leq c_{4}|y|^{-d} \Phi\left(\delta_{\mathbb{H}}(y)\right)^{1 / 2} \leq c_{5} G_{\mathbb{H}}\left(x_{0}, y\right) .
$$

We have proved (7.6) for $\mathbb{H}$.

Step 2. We assume $\delta_{D}(x) \wedge \delta_{D}(y) \geq 3$. Then using the monotonicity of Green functions (7.3) and Step 1, we have

$$
\begin{aligned}
\frac{G_{D}(x, y)}{G_{D}(x, w)} & \leq \frac{G_{\mathbb{H}}(x, y)}{G_{\mathbb{H}_{1}}(x, w)} \leq c_{6} \frac{G_{\mathbb{H}}(x, y)}{G_{\mathbb{H}}(x, w)} \leq c_{7} \frac{G_{\mathbb{H}}\left(x_{0}, y\right)}{G_{\mathbb{H}}\left(x_{0}, w\right)} \\
& \leq c_{7} c_{6}^{-1} \frac{G_{\mathbb{H}_{1}}\left(x_{0}, y\right)}{G_{\mathbb{H}}\left(x_{0}, w\right)} \leq c_{7} c_{6}^{-1} \frac{G_{D}\left(x_{0}, y\right)}{G_{D}\left(x_{0}, w\right)} .
\end{aligned}
$$

Step 3. Let

$$
x_{1}:=\left\{\begin{array}{ll}
x & \text { if } \delta_{D}(x)>3, \\
(\widetilde{x}, 3) & \text { if } \delta_{D}(x) \leq 3,
\end{array} \quad \text { and } \quad y_{1}:= \begin{cases}y & \text { if } \delta_{D}(y)>3 \\
(\widetilde{y}, 3), & \text { if } \delta_{D}(y) \leq 3\end{cases}\right.
$$

We use Theorem 4.3 when $\delta_{D}(x) \leq 3$ and get

$$
G_{D}(x, w)=\frac{G_{D}(x, w)}{G_{D}\left(x, x_{0}\right)} G_{D}\left(x_{0}, x\right) \geq c_{11} \frac{G_{D}\left(x_{1}, w\right)}{G_{D}\left(x_{1}, x_{0}\right)} G_{D}\left(x_{0}, x\right) .
$$

Since $|x-y| \geq \frac{3}{4}|y| \geq \frac{15}{2}$, we use Theorem 4.3 when $\delta_{D}(x) \leq 3$ and get

$$
G_{D}(x, y)=\frac{G_{D}(x, y)}{G_{D}\left(x, x_{0}\right)} G_{D}\left(x_{0}, x\right) \leq c_{12} \frac{G_{D}\left(x_{1}, y\right)}{G_{D}\left(x_{1}, x_{0}\right)} G_{D}\left(x_{0}, x\right) .
$$

If $\delta_{D}(x) \leq 3$, then $\left|y-x_{1}\right| \geq|y-x|-\left|x-x_{1}\right| \geq|y-x|-3 \geq \frac{9}{2}$. Thus using Theorem 4.3 again when $\delta_{D}(y) \leq 3$, we get

$$
G_{D}\left(x_{1}, y\right)=\frac{G_{D}\left(y, x_{1}\right)}{G_{D}\left(y, x_{0}\right)} G_{D}\left(y, x_{0}\right) \leq c_{13} \frac{G_{D}\left(y_{1}, x_{1}\right)}{G_{D}\left(y_{1}, x_{0}\right)} G_{D}\left(y, x_{0}\right) .
$$

From (7.9)-(7.10),

$$
G_{D}(x, y) \leq c_{14} \frac{G_{D}\left(x_{1}, y_{1}\right)}{G_{D}\left(x_{1}, x_{0}\right)} G_{D}\left(x_{0}, x\right) \frac{G_{D}\left(y, x_{0}\right)}{G_{D}\left(y_{1}, x_{0}\right)} .
$$


Combining (7.8) and (7.11) and using Step 2, we conclude that

$$
\begin{aligned}
\frac{G_{D}(x, y)}{G_{D}(x, w)} & \leq c_{15} \frac{G_{D}\left(x_{1}, y_{1}\right)}{G_{D}\left(x_{1}, w\right)} \frac{G_{D}\left(x_{0}, y\right)}{G_{D}\left(x_{0}, y_{1}\right)} \\
& =c_{15}\left(\frac{G_{D}\left(x_{1}, y_{1}\right)}{G_{D}\left(x_{1}, w\right)} \frac{G_{D}\left(x_{0}, w\right)}{G_{D}\left(x_{0}, y_{1}\right)}\right) \frac{G_{D}\left(x_{0}, y\right)}{G_{D}\left(x_{0}, w\right)} \\
& \leq c_{16} \frac{G_{D}\left(x_{0}, y\right)}{G_{D}\left(x_{0}, w\right)} .
\end{aligned}
$$

For $E \subset D$ and $n \geq 1$, define $E^{n}=E \cap\left\{x \in D: 2^{n} \leq|x|<2^{n+1}\right\}$.

Proposition 7.3. The set $E$ is minimally thin in $D$ at infinity with respect to $X$ if and only if

$$
\sum_{n=1}^{\infty} R_{M_{D}(\cdot, \infty)}^{E^{n}}\left(x_{0}\right)<\infty
$$

Proof. Assume (7.12) holds. Then there exists $n_{0} \in \mathbb{N}$ such that $\sum_{n_{0}}^{\infty} R_{M_{D}(\cdot, \infty)}^{E^{n}}\left(x_{0}\right)$ $<\frac{1}{2} M_{D}\left(x_{0}, \infty\right)$. Let $B=\bar{B}\left(0,2^{n_{0}}\right)$. Then $A:=B^{c} \cap E \subset \bigcup_{n_{0}}^{\infty} E^{n}$. Therefore,

$$
R_{M_{D}(\cdot, \infty)}^{E}\left(x_{0}\right) \leq \sum_{n_{0}}^{\infty} R_{M_{D}(\cdot, \infty)}^{E^{n}}\left(x_{0}\right)<\frac{1}{2} M_{D}\left(x_{0}, \infty\right),
$$

implying $\widehat{R}_{M_{D}(\cdot, \infty)}^{E}\left(x_{0}\right)<\frac{1}{2} M_{D}\left(x_{0}, \infty\right)$. Hence, $E$ is minimally thin in $D$ at infinity with respect to $X$.

Conversely, suppose that $E$ is minimally thin in $D$ at infinity with respect to $X$. By Proposition 6.2, there exists a potential $u$ such that

$$
\liminf _{x \rightarrow \infty, x \in E} \frac{u(x)}{M_{D}(x, \infty)}=+\infty .
$$

Without loss of generality, we may assume that $u\left(x_{0}\right) \leq\left(2 C_{7}\right)^{-1}$, and $C_{7}$ is the constant from Lemma 7.2. There exists $n_{1} \in \mathbb{N}$ with $n_{1} \geq 10$ such that $u(x)>$ $M_{D}(x, \infty)$ for all $x \in E \cap \bar{B}\left(0,2^{n_{1}}\right)^{c}$. Thus, $E \subset B\left(0,2^{n_{1}}\right) \cap\left\{u>M_{D}(\cdot, \infty)\right\}$. For $n \geq n_{1}$ define

$$
F_{n}=\left\{x \in D: 2^{n} \leq|x|<2^{n+1}, u(x)>M_{D}(x, \infty)\right\} \quad \text { and } \quad F=\bigcup_{n_{1}}^{\infty} F_{n} .
$$

Let $x \in E^{n}$. Since $|x|>2^{n_{1}}$, we have that $u(x)>M_{D}(x, \infty)$ and thus $x \in$ $F_{n}$. This shows that $E^{n} \subset F_{n}, n \geq n_{1}$. Therefore, it suffices to show that $\sum_{n_{1}}^{\infty} R_{M_{D}(\cdot, \infty)}^{F_{n}}\left(x_{0}\right)<\infty$. Since $u>M_{D}(\cdot, \infty)$ on $F$, it follows that $R_{M_{D}(\cdot, \infty)}^{F_{n}}\left(x_{0}\right) \leq$ $u\left(x_{0}\right) \leq c$.

Let $i \in\{1,2,3\}$. For every $n \in \mathbb{N}$, let $U_{n}=F_{n_{1}+3 n+i}$. Since $i \in\{1,2,3\}$ is arbitrary, it suffices to show that $\sum_{n=1}^{\infty} R_{M_{D}(\cdot, \infty)}^{U_{n}}\left(x_{0}\right)<\infty$. Let $U=\bigcup_{n=1}^{\infty} U_{n}$. Then $U \subset F$ and thus $R_{M_{D}(\cdot, \infty)}^{U}\left(x_{0}\right) \leq u\left(x_{0}\right)$. Note that since $U$ is open, $\widehat{R}_{M_{D}(\cdot, \infty)}^{U}=$ $R_{M_{D}(\cdot, \infty)}^{U}$ (see [6, p. 205]). Since $u$ is a potential, the same holds for $\widehat{R}_{M_{D}(\cdot, \infty)}^{U}$; hence 
there exists a measure $\mu$ such that $R_{M_{D}(\cdot, \infty)}^{U}=G_{D} \mu$. Moreover, since $R_{M_{D}(\cdot, \infty)}^{U}$ is harmonic on $\bar{U}^{c}$ (cf. [6, III.2.5]), $\mu\left(U^{c}\right)=0$. Let $\mu_{n}:=\mu_{\mid \bar{U}_{n}}$. Since $\bar{U}_{n}$ are pairwise disjoint,

$$
\mu=\sum_{n=1}^{\infty} \mu_{n} \quad \text { and } \quad G_{D} \mu=\sum_{n=1}^{\infty} G_{D} \mu_{n} .
$$

Fix $n \in \mathbb{N}$ and consider $l \in \mathbb{N}, x \in U_{n}, y \in \bar{U}_{l}$. If $l>n$, then $|x|<2^{n_{1}+3 n+i+1} \leq$ $2^{1-3(l-n)}|y| \leq \frac{1}{4}|y|$, and hence $|x-y| \geq|y|-|x| \geq \frac{3}{4}|y|$. If $l<n$, then analogously, $|y| \leq \frac{1}{4}|x|$, hence $|x-y| \geq|x|-|y| \geq \frac{3}{4}|x| \geq \frac{3}{4}|y|$. Thus, in both cases, $|x-y| \geq \frac{3}{4}|y|$ for every $x \in U_{n}$ and $y \in \overline{U_{l}}, l \neq n$.

Define $\mu_{n}^{\prime}=\mu-\mu_{n}$ and let $x \in U_{n}$. By Lemma 7.2

$$
\begin{aligned}
& G_{D} \mu_{n}^{\prime}(x)=\int_{D} G_{D}(x, y) \mu_{n}^{\prime}(d y) \leq C_{7} M_{D}(x, \infty) \int_{D} G_{D}\left(x_{0}, y\right) \mu_{n}^{\prime}(d y) \\
& \leq C_{5} M_{D}(x, \infty) G_{D} \mu\left(x_{0}\right) \leq C_{7} M_{D}(x, \infty) u\left(x_{0}\right)<\frac{1}{2} M_{D}(x, \infty) .
\end{aligned}
$$

Since $G_{D} \mu_{n}+G_{D} \mu_{n}^{\prime}=G_{D} \mu=R_{M_{D}(\cdot, \infty)}^{U}=M_{D}(\cdot, \infty)$ on $U$, it follows that $G_{D} \mu_{n}=$ $M_{D}(\cdot, \infty)-G_{D} \mu_{n}^{\prime} \geq M_{D}(\cdot, \infty)-\frac{1}{2} M_{D}(\cdot, \infty)=\frac{1}{2} M_{D}(\cdot, \infty)$ on $U_{n}$. This implies that $G_{D} \mu_{n} \geq \frac{1}{2} R_{M_{D}(\cdot, \infty)}^{U_{n}}$. Finally,

$$
\sum_{n=1}^{\infty} R_{M_{D}(\cdot, \infty)}^{U_{n}}\left(x_{0}\right) \leq 2 \sum_{n=1}^{\infty} G_{D} \mu_{n}\left(x_{0}\right)=2 G_{D} \mu\left(x_{0}\right)<\infty .
$$

Lemma 7.4. There exists $c>1$ such that

$$
c^{-1} G_{D}\left(x, x_{0}\right)|x|^{d} \leq M_{D}(x, \infty) \leq c G_{D}\left(x, x_{0}\right)|x|^{d} \quad x \in B(0,30)^{c} \cap D .
$$

Proof. Step 1. Assume $\delta_{D}(x) \geq 3$ and $|x| \geq 10$. For $w \in B(0,10|x|)^{c} \cap D$ with $\delta_{D}(w) \geq 3$, using the monotonicity of Green functions and (7.3),

$$
\frac{G_{D}(x, w)}{G_{D}\left(x_{0}, w\right) G_{D}\left(x, x_{0}\right)} \asymp \frac{G_{\mathbb{H}}(x, w)}{G_{\mathbb{H}}\left(x_{0}, w\right) G_{\mathbb{H}}\left(x, x_{0}\right)} .
$$

Thus by (7.2),

$$
\frac{M_{D}(x, \infty)}{G_{D}\left(x, x_{0}\right)} \asymp \frac{M_{\mathbb{H}}(x, \infty)}{G_{\mathbb{H}}\left(x, x_{0}\right)} \asymp \frac{\Phi\left(\delta_{\mathbb{H}}(x)\right)^{1 / 2}}{G_{\mathbb{H}}\left(x, x_{0}\right)} .
$$

Now (7.13) follows from (7.14) and (7.4) immediately.

Step 2. Assume $\delta_{D}(x) \leq 3$ and $|x| \geq 30$. Let $x_{1}:=(\widetilde{x},|x| / 3)$ so that $x, x_{1} \in$ $B((\widetilde{x}, 0),|x| / 2) \cap D$ and $x_{0} \notin B((\widetilde{x}, 0), 2|x| / 3) \cap D$. In fact, $\left|x_{0}-(\widetilde{x}, 0)\right| \geq|(\widetilde{x}, 0)|-$ $5 \geq|x|-\left|x_{d}\right|-5 \geq|x|-9 \geq 2|x| / 3$. Thus by Theorem 4.3 , we have that for $w \in B(0,10|x|)^{c} \cap D$ with $\delta_{D}(w) \geq 3$,

$$
\frac{G_{D}(x, w)}{G_{D}\left(x, x_{0}\right)} \asymp \frac{G_{D}\left(x_{1}, w\right)}{G_{D}\left(x_{1}, x_{0}\right)},
$$

which implies that

$$
\frac{M_{D}(x, \infty)}{G_{D}\left(x, x_{0}\right)} \asymp \frac{M_{D}\left(x_{1}, \infty\right)}{G_{D}\left(x_{1}, x_{0}\right)} .
$$


Moreover, since $|x| / 3 \leq\left|x_{1}\right| \leq 2|x|$, by Step 1 and (7.15),

$$
\frac{M_{D}(x, \infty)}{G_{D}\left(x, x_{0}\right)} \asymp \frac{M_{D}\left(x_{1}, \infty\right)}{G_{D}\left(x_{1}, x_{0}\right)} \asymp\left|x_{1}\right|^{-d} \asymp|x|^{-d} .
$$

We have proved the lemma.

Let $g(x):=G_{D}\left(x, x_{0}\right) \wedge C_{8}$ where $C_{8}:=C_{5}\left(\sup _{s>25} \Phi(s) s^{-d}\right)>0$ so that, by (4.2), $g(x)=G_{D}\left(x, x_{0}\right)$ for every $x \in B(0,30)^{c} \cap D$. Lemma 7.4 implies that for $n \geq 5$,

$$
\begin{aligned}
c_{1}^{-1} 2^{n d} g(x) & =c_{1}^{-1} 2^{n d} G_{D}\left(x, x_{0}\right) \leq M_{D}(x, \infty) \\
& \leq c_{1} 2^{n d} G_{D}\left(x, x_{0}\right)=c_{1} 2^{n d} g(x), \quad x \in E^{n} .
\end{aligned}
$$

This implies that for $n \geq 5, c_{1}^{-1} 2^{n d} R_{g}^{E^{n}} \leq R_{M_{D}(\cdot, \infty)}^{E^{n}} \leq c_{1} 2^{n d} R_{g}^{E^{n}}$. In particular,

$$
\sum_{n=1}^{\infty} R_{M_{D}(\cdot, \infty)}^{E^{n}}\left(x_{0}\right)<\infty \quad \text { if and only if } \sum_{n=1}^{\infty} 2^{n d} R_{g}^{E^{n}}\left(x_{0}\right)<\infty .
$$

Note that $\widehat{R}_{g}^{E^{n}}$ is a potential; hence there exists a measure $\lambda_{n}$ (supported by $\left.\bar{E}_{n}\right)$ such that $\widehat{R}_{g}^{E^{n}}=G_{D} \lambda_{n}$. Also, $\widehat{R}_{g}^{E^{n}}(\cdot)=g(\cdot)=G_{D}\left(\cdot, x_{0}\right)$ on $\overline{E^{n}}$ for $n \geq 5$, hence

$$
\begin{aligned}
\widehat{R}_{g}^{E^{n}}\left(x_{0}\right) & =\int_{\overline{E^{n}}} g(y) \lambda_{n}(d y)=\int_{\bar{E}_{n}} \widehat{R}_{g}^{E^{n}}(y) \lambda_{n}(d y) \\
& =\int_{D} \int_{D} G_{D}(x, y) \lambda_{n}(d y) \lambda_{n}(d x)=\gamma_{g}\left(E^{n}\right) .
\end{aligned}
$$

We conclude from (7.16) that

$$
\sum_{n=1}^{\infty} R_{M_{D}(\cdot, \infty)}^{E^{n}}\left(x_{0}\right)<\infty \quad \text { if and only if } \quad \sum_{n=1}^{\infty} 2^{n d} \gamma_{g}\left(E^{n}\right)<\infty .
$$

Thus we have proved the following Wiener-type criterion for minimal thinness.

Corollary 7.5. The set $E \subset D$ is minimally thin in $D$ at infinity with respect to $X$ if and only if $\sum_{n=1}^{\infty} 2^{\text {nd }} \gamma_{g}\left(E^{n}\right)<\infty$.

Remark 7.6. Note that [2, Part I, 11.3, p. 71] has a similar criterion (attributed to Lelong-Ferrand) in case when $D$ is the half-space $\mathbb{H}$ and $X$ is Brownian motion: $E$ is minimally thin at infinity if and only if $\sum_{n=1}^{\infty} 2^{-n d} \gamma\left(E^{n}\right)<\infty$. Here $\gamma\left(E^{n}\right)=$ $\gamma_{V}\left(E^{n}\right)$ is the Green energy defined with respect to the function $V(x)=x_{d}$ (and not $g(x))$; see [2, Part I, p. 66].

Finally, we prove a version of Aikawa's criterion for minimal thinness.

Proposition 7.7. Suppose that $\left(\mathcal{E}, \mathcal{F}_{D}\right)$ satisfies the Hardy inequality. Let $\left\{Q_{j}\right\}_{j \geq 1}$ be a Whitney decomposition of $D, E \subset D$, and let $x_{j}$ denote the center of $Q_{j}$. Then $E$ is minimally thin in $D$ at infinity with respect to $X$ if and only if

$$
\sum_{j: Q_{j} \subset B\left(0,2^{5}\right)^{c}} \operatorname{dist}\left(0, Q_{j}\right)^{d} G_{D}\left(x_{j}, x_{0}\right)^{2} \operatorname{Cap}_{D}\left(E \cap Q_{j}\right)<\infty .
$$


Proof. It follows from Corollary 7.5 that $E$ is minimally thin in $D$ at infinity with respect to $X$ if and only if $\sum_{n=1}^{\infty} 2^{n d} \gamma_{g}\left(E^{n}\right)<\infty$. Further, let $V_{n}=\left\{x \in \mathbb{R}^{d}\right.$ : $\left.2^{n} \leq|x|<2^{n+1}\right\}$ so that $E^{n}=E \cap V_{n}$. If $V_{n} \cap Q_{j} \neq \emptyset$, then $\operatorname{dist}\left(0, Q_{j}\right) \asymp 2^{n}$. By the quasi-additivity of $\gamma_{g}$ (Proposition $5.11(2)$ ),

$$
\begin{aligned}
\sum_{n=1}^{\infty} 2^{n d} \gamma_{g}\left(E^{n}\right) & \asymp \sum_{n=1}^{\infty} 2^{n d} \sum_{j: Q_{j} \subset B\left(0,2^{5}\right)^{c}} \gamma_{g}\left(E^{n} \cap Q_{j}\right) \\
& \asymp \sum_{j: Q_{j} \subset B\left(0,2^{5}\right)^{c}} \sum_{n, V_{n} \cap Q_{j} \neq \emptyset} \operatorname{dist}\left(0, Q_{j}\right)^{d} \gamma_{g}\left(E^{n} \cap Q_{j}\right) \\
& =\sum_{j: Q_{j} \subset B\left(0,2^{5}\right)^{c}} \operatorname{dist}\left(0, Q_{j}\right)^{d} \sum_{n, V_{n} \cap Q_{j} \neq \emptyset} \gamma_{g}\left(E^{n} \cap Q_{j}\right) \\
& \asymp \sum_{j: Q_{j} \subset B\left(0,2^{5}\right)^{c}} \operatorname{dist}\left(0, Q_{j}\right)^{d} \gamma_{g}\left(E \cap Q_{j}\right) .
\end{aligned}
$$

For the last line we argue as follows: One inequality is the subadditivity of capacity. For the other note that there exists $N \in \mathbb{N}$ such that for every $Q_{j}, \sum_{n, V_{n} \cap Q_{j} \neq \emptyset} 1=$ $\sum_{n} 1_{V_{n} \cap Q_{j}} \leq N$. Hence, $\sum_{n, V_{n} \cap Q_{j} \neq \emptyset} \gamma_{g}\left(E \cap V_{n} \cap Q_{j}\right) \leq \sum_{n, V_{n} \cap Q_{j} \neq \emptyset} \gamma_{g}\left(E \cap Q_{j}\right) \leq$ $N \gamma_{g}\left(E \cap Q_{j}\right)$.

Finally, since $g$ satisfies the scale invariant inequality (5.12), it follows from Lemma $5.8(2)$ that $\gamma_{g}\left(E \cap Q_{j}\right) \asymp g\left(x_{j}\right)^{2} \operatorname{Cap}_{D}\left(E \cap Q_{j}\right)$. Since $g(x)=G_{D}\left(x, x_{0}\right)$ for $x \in B(0,30)^{c} \cap D$, this completes the proof.

Proof of Theorem 1.7. Assume that $E$ is minimally thin at $\infty$. By Proposition 7.7 .

$$
\sum_{j: Q_{j} \subset B\left(0,2^{5}\right)^{c}} \operatorname{dist}\left(0, Q_{j}\right)^{d} G_{D}\left(x_{j}, x_{0}\right)^{2} \operatorname{Cap}_{D}\left(E \cap Q_{j}\right)<\infty .
$$

First note that

$$
\operatorname{Cap}_{D}\left(E \cap Q_{j}\right) \geq c_{1} \sigma_{1}\left(E \cap Q_{j}\right)=c_{1} \int_{E} \mathbf{1}_{Q_{j}}(x) \Psi\left(\delta_{D}(x)^{-1}\right) d x .
$$

Next, $\operatorname{dist}\left(0, Q_{j}\right) \asymp|x|$ for $x \in Q_{j}$. Therefore,

$$
\begin{aligned}
& \sum_{j: Q_{j} \subset B\left(0,2^{5}\right)^{c}} \operatorname{dist}\left(0, Q_{j}\right)^{d} G_{D}\left(x_{j}, x_{0}\right)^{2} \operatorname{Cap}_{D}\left(E \cap Q_{j}\right) \\
\geq & c_{2} \int_{E}|x|^{d} G_{D}\left(x, x_{0}\right)^{2} \Psi\left(\delta_{D}(x)^{-1}\right) \sum_{j: Q_{j} \subset B\left(0,2^{5}\right)^{c}} \mathbf{1}_{Q_{j}}(x) d x \\
\geq & c_{2} \int_{E \cap B\left(0,2^{8}\right)^{c}}|x|^{d} G_{D}\left(x, x_{0}\right)^{2} \Psi\left(\delta_{D}(x)^{-1}\right) d x .
\end{aligned}
$$

Conversely, assume that $E$ is the union of a subfamily of Whitney cubes. Then $E \cap$ $Q_{j}$ is either empty or equal to $Q_{j}$. Since $\operatorname{Cap}_{D}\left(Q_{j}\right) \asymp \sigma_{1}\left(Q_{j}\right)=\int_{Q_{j}} \Psi\left(\delta_{D}(x)^{-1}\right) d x$, we can reverse the first inequality in the display above to conclude that

$$
\begin{aligned}
& \sum_{j: Q_{j} \subset B\left(0,2^{5}\right)^{c}} \operatorname{dist}\left(0, Q_{j}\right)^{-d} G_{D}\left(x_{j}, x_{0}\right)^{2} \operatorname{Cap}_{D}\left(E \cap Q_{j}\right) \\
& \leq c_{3} \int_{E \cap B\left(0,2^{5}\right)^{c}}|x|^{d} G_{D}\left(x, x_{0}\right)^{2} \Psi\left(\delta_{D}(x)^{-1}\right) d x .
\end{aligned}
$$


Proof of Corollary 1.8. By integrating the heat kernel estimates in 10, Theorem 5.8], one can easily get that, for $x \in B(0,10)^{c} \cap D$,

$$
\begin{aligned}
G_{D}\left(x, x_{0}\right) & \asymp \frac{\Phi\left(\left|x-x_{0}\right|\right)}{\left|x-x_{0}\right|^{d}}\left(1 \wedge \frac{\Phi\left(\delta_{D}(x)\right)}{\Phi\left(\left|x-x_{0}\right|\right)}\right)^{1 / 2}\left(1 \wedge \frac{\Phi\left(\delta_{D}\left(x_{0}\right)\right)}{\Phi\left(\left|x-x_{0}\right|\right)}\right)^{1 / 2} \\
& \asymp \frac{\Phi\left(\delta_{D}(x)\right)^{1 / 2}}{|x|^{d}}
\end{aligned}
$$

(see the proof of [14, Theorem 7.3(iv)]). Thus the corollary immediately follows from this and Theorem 1.7 .

Remark 7.8. Note that by using (17.18) we have the following sharp two-sided Martin function estimates for half-space-like $C^{1,1}$ open set $D$ : for every $z \in \partial D$,

$$
M_{D}(x, z) \asymp \frac{\Phi\left(\delta_{D}(x)\right)^{1 / 2}\left|x_{0}-z\right|^{d}}{|x-z|^{d}}
$$

and

$$
M_{D}(x, \infty) \asymp \Phi\left(\delta_{D}(x)\right)^{1 / 2}
$$

\section{Minimal thinness of A SET Under the GRAPH OF A FUnCtion}

In this section, we will study minimal thinness of a set below the graph of a Lipschitz function, both for finite and infinite boundary points. We start by recalling Burdzy's result; cf. [11,25].

Let $f: \mathbb{R}^{d-1} \rightarrow[0, \infty)$ be a Lipschitz function. The set $A=\left\{x=\left(\widetilde{x}, x_{d}\right) \in \mathbb{H}\right.$ : $\left.0<x_{d} \leq f(\widetilde{x})\right\}$ is minimally thin in $\mathbb{H}$ with respect to Brownian motion at $z=0$ if and only if

$$
\int_{\{|\widetilde{x}|<1\}} f(\widetilde{x})|\widetilde{x}|^{-d} d \widetilde{x}<\infty .
$$

It was shown recently in 28 that the same criterion for minimal thinness is true for the subordinate Brownian motions studied there. By using Corollary 1.5 one can follow the proof of [28, Theorem 4.4] (cf. also the proof of Theorem 8.2 below) and show that the following Burdzy criterion for minimal thinness holds.

Proposition 8.1. Assume that $D:=\left\{x=\left(\widetilde{x}, x_{d}\right) \in \mathbb{R}^{d}: x_{d}>h(\widetilde{x})\right\}$ is the domain above the graph of a bounded $C^{1,1}$ function $h$ and that $f: \mathbb{R}^{d-1} \rightarrow[0, \infty)$ is a Lipschitz function. Suppose either $h \equiv 0$ or $\gamma=1$ in (1.2). Then the set $A:=\left\{x=\left(\widetilde{x}, x_{d}\right) \in D: h(\widetilde{x})<x_{d} \leq f(\widetilde{x})+h(\widetilde{x})\right\}$ is minimally thin in $D$ at $z=(\widetilde{0}, h(\widetilde{0}))$ with respect to $X$ if and only if (8.1) holds

We omit the proof and concentrate on a similar question for minimal thinness at infinity.

Theorem 8.2. Suppose that (H2) holds and $\gamma=1$ in (1.2), and let $D=\{x=$ $\left.\left(\widetilde{x}, x_{d}\right) \in \mathbb{R}^{d}: x_{d}>h(\widetilde{x})\right\}$ be the domain above the graph of a bounded $C^{1,1}$ function $h$. Let $f: \mathbb{R}^{d-1} \rightarrow[0, \infty)$ be a Lipschitz function. Then the set $A:=\left\{x=\left(\widetilde{x}, x_{d}\right) \in\right.$ $\left.\mathbb{R}^{d}: h(\widetilde{x})<x_{d} \leq f(\widetilde{x})+h(\widetilde{x})\right\}$ is minimally thin in $D$ at infinity with respect to $X$ if and only if

$$
\int_{\{|\widetilde{x}|>1\}} f(\widetilde{x})|\widetilde{x}|^{-d} d \widetilde{x}<\infty .
$$


Proof. Without loss of generality we may assume that $f(\widetilde{x})=0$ for $|\widetilde{x}| \leq 1, h(\widetilde{0})=0$ and $A=\left\{x=\left(\widetilde{x}, x_{d}\right) \in \mathbb{R}^{d}:|\widetilde{x}|>1, h(\widetilde{x})<x_{d} \leq f(\widetilde{x})+h(\widetilde{x})\right\}$. We first note that by the Lipschitz continuity of $f$ and boundedness of $h$, it follows that $|\widetilde{x}| \leq|x| \leq c_{1}|\widetilde{x}|$ for $x=\left(\widetilde{x}, x_{d}\right) \in A$. Hence by Fubini's theorem we have

$$
\begin{aligned}
\int_{A}|x|^{-d} d x & =\int_{|\widetilde{x}|>1} d \widetilde{x} \int \mathbf{1}_{A}\left(\widetilde{x}, x_{d}\right)|x|^{-d} d x_{d} \\
& \asymp \int_{|\widetilde{x}|>1}|\widetilde{x}|^{-d} d \widetilde{x} \int_{h(\widetilde{x})}^{f(\widetilde{x})+h(\widetilde{x})} d x_{d} \\
& =\int_{|\widetilde{x}|>1} f(\widetilde{x})|\widetilde{x}|^{-d} d \widetilde{x} .
\end{aligned}
$$

It follows from Corollary [1.8(i) that if $A$ is minimally thin in $D$ at infinity, then (8.2) holds true.

For the converse, let $\left\{Q_{j}\right\}$ be a Whitney decomposition of $D$ and define $E=$ $\bigcup_{Q_{j} \cap A \neq \emptyset} Q_{j}$; clearly $A \subset E$. Let $Q_{j}^{*}$ be the interior of the double of $Q_{j}$ and note that $\left\{Q_{j}^{*}\right\}$ has bounded multiplicity, say $N$. Moreover, if $Q_{j} \cap A \neq \emptyset$, then by the Lipschitz continuity of $f$ and $h$ we have $\left|Q_{j}^{*} \cap A\right| \asymp\left|Q_{j}\right|$. Moreover, for $x \in Q_{j}^{*}$ we have $|x| \asymp \operatorname{dist}\left(0, Q_{j}\right)$. Therefore

$$
\begin{aligned}
\int_{A}|x|^{-d} d x & \leq \int_{E}|x|^{-d} d x=\sum_{Q_{j} \cap A \neq \emptyset} \int_{Q_{j}}|x|^{-d} d x \\
& \leq c_{2} \sum_{Q_{j} \cap A \neq \emptyset}\left|Q_{j}^{*} \cap A\right| \operatorname{dist}\left(0, Q_{j}\right)^{-d} \\
& \leq c_{3} \sum_{Q_{j} \cap A \neq \emptyset} \int_{Q_{j}^{*} \cap A}|x|^{-d} d x \leq c_{3} N \int_{A}|x|^{-d} d x .
\end{aligned}
$$

If (8.2) holds, then (8.3) and (8.4) imply that $\int_{E}|x|^{-d} d x<\infty$. Hence, by Corollary 1.8 (ii), $E$ is minimally thin, and thus $A$ is also minimally thin.

Example 8.3. Suppose that $c>0$ and $\delta \geq 0$. By Theorem 8.2 the set $A:=$ $\left\{x=\left(\widetilde{x}, x_{d}\right) \in \mathbb{H}: 0<x_{d} \leq c|\widetilde{x}|^{1-\delta}\right\}$ is minimally thin in $\mathbb{H}$ at infinity with respect to $X$ if and only if $\delta>0$.

\section{ACKNOWLEDGEMENTS}

The authors are grateful to the referee for helpful comments, and in particular for suggesting a simpler (and better) version of Theorem 8.2 and providing its proof.

\section{REFERENCES}

[1] H. Aikawa, Quasiadditivity of capacity and minimal thinness, Ann. Acad. Sci. Fenn. Ser. A I Math. 18 (1993), no. 1, 65-75. MR 1207895 (94d:31004)

[2] H. Aikawa and M. Essén, Potential theory-selected topics, Lecture Notes in Mathematics, vol. 1633, Springer-Verlag, Berlin, 1996. MR1439503 (98f:31005)

[3] H. Aikawa, T. Kilpeläinen, N. Shanmugalingam, and X. Zhong, Boundary Harnack principle for p-harmonic functions in smooth Euclidean domains, Potential Anal. 26 (2007), no. 3, 281-301, DOI 10.1007/s11118-006-9036-y. MR2286038(2008a:31012)

[4] D. H. Armitage and S. J. Gardiner, Classical potential theory, Springer Monographs in Mathematics, Springer-Verlag London, Ltd., London, 2001. MR.1801253 (2001m:31001)

[5] A. Beurling, A minimum principle for positive harmonic functions, Ann. Acad. Sci. Fenn. Ser. A I No. 372 (1965), 7. MR0188466 (32 \#5904) 
[6] J. Bliedtner and W. Hansen, Potential theory: An analytic and probabilistic approach to balayage, Universitext, Springer-Verlag, Berlin, 1986. MR850715 (88b:31002)

[7] K. Bogdan, Sharp estimates for the Green function in Lipschitz domains, J. Math. Anal. Appl. 243 (2000), no. 2, 326-337, DOI 10.1006/jmaa.1999.6673. MR.1741527 (2001b:31007)

[8] K. Bogdan, T. Grzywny, and M. Ryznar, Density and tails of unimodal convolution semigroups, J. Funct. Anal. 266 (2014), no. 6, 3543-3571, DOI 10.1016/j.jfa.2014.01.007. MR3165234

[9] K. Bogdan, T. Grzywny, and M. Ryznar, Barriers, exit time and survival probability for unimodal Lévy processes, Probab. Theory Related Fields 162 (2015), no. 1-2, 155-198, DOI 10.1007/s00440-014-0568-6. MR 3350043

[10] K. Bogdan, T. Grzywny, and M. Ryznar, Dirichlet heat kernel for unimodal Lévy processes, Stochastic Process. Appl. 124 (2014), no. 11, 3612-3650, DOI 10.1016/j.spa.2014.06.001. MR 3249349

[11] K. Burdzy, Brownian excursions and minimal thinness. I, Ann. Probab. 15 (1987), no. 2, 676-689. MR885137 (88h:60149)

[12] Z.-Q. Chen and P. Kim, Global Dirichlet heat kernel estimates for symmetric Lévy processes in half-space, Preprint, arXiv:1504.04673.

[13] Z.-Q. Chen, P. Kim, and T. Kumagai, On heat kernel estimates and parabolic Harnack inequality for jump processes on metric measure spaces, Acta Math. Sin. (Engl. Ser.) 25 (2009), no. 7, 1067-1086, DOI 10.1007/s10114-009-8576-7. MR2524930(2011b:60334)

[14] Z.-Q. Chen, P. Kim, and R. Song, Dirichlet heat kernel estimates for rotationally symmetric Lévy processes, Proc. Lond. Math. Soc. (3) 109 (2014), no. 1, 90-120, DOI $10.1112 / \mathrm{plms} / \mathrm{pdt068}$. MR 3237737

[15] Z.-Q. Chen and T. Kumagai, Heat kernel estimates for jump processes of mixed types on metric measure spaces, Probab. Theory Related Fields 140 (2008), no. 1-2, 277-317, DOI 10.1007/s00440-007-0070-5. MR2357678 (2009e:60186)

[16] K. L. Chung, Lectures from Markov processes to Brownian motion, Grundlehren der Mathematischen Wissenschaften [Fundamental Principles of Mathematical Science], vol. 249, Springer-Verlag, New York-Berlin, 1982. MR648601 (84c:60091)

[17] B. Dyda and A. V. Vähäkangas, A framework for fractional Hardy inequalities, Ann. Acad. Sci. Fenn. Math. 39 (2014), no. 2, 675-689, DOI 10.5186/aasfm.2014.3943. MR3237044

[18] B. Dahlberg, A minimum principle for positive harmonic functions, Proc. London Math. Soc. (3) 33 (1976), no. 2, 238-250. MR0409847 (53 \#13599)

[19] J. L. Doob, Classical potential theory and its probabilistic counterpart, Grundlehren der Mathematischen Wissenschaften [Fundamental Principles of Mathematical Sciences], vol. 262, Springer-Verlag, New York, 1984. MR731258 (85k:31001)

[20] M. Essén, On Wiener conditions for minimally thin and rarefied sets, Complex analysis, Birkhäuser, Basel, 1988, pp. 41-50. MR981400(89m:31005)

[21] L. C. Evans and R. F. Gariepy, Measure theory and fine properties of functions, Studies in Advanced Mathematics, CRC Press, Boca Raton, FL, 1992. MR 1158660 (93f:28001)

[22] H. Föllmer, Feine Topologie am Martinrand eines Standardprozesses (German, with English summary), Z. Wahrscheinlichkeitstheorie und Verw. Gebiete 12 (1969), 127-144. MR0245092 (39 \#6404)

[23] B. Fuglede, On the theory of potentials in locally compact spaces, Acta Math. 103 (1960), 139-215. MR0117453 (22 \#8232)

[24] B. Fuglede, Le théorème du minimax et la théorie fine du potentiel (French), Ann. Inst. Fourier (Grenoble) 15 (1965), no. fasc. 1, 65-88. MR0190368 (32 \#7781)

[25] S. J. Gardiner, A short proof of Burdzy's theorem on the angular derivative, Bull. London Math. Soc. 23 (1991), no. 6, 575-579, DOI 10.1112/blms/23.6.575. MR.1135189 (93a:31006)

[26] T. Grzywny, On Harnack inequality and Hölder regularity for isotropic unimodal Lévy processes, Potential Anal. 41 (2014), no. 1, 1-29, DOI 10.1007/s11118-013-9360-y. MR3225805

[27] P. Kim and A. Mimica, Green function estimates for subordinate Brownian motions: stable and beyond, Trans. Amer. Math. Soc. 366 (2014), no. 8, 4383-4422, DOI 10.1090/S00029947-2014-06017-0. MR.3206464

[28] P. Kim, R. Song, and Z. Vondraček, Minimal thinness for subordinate Brownian motion in half-space (English, with English and French summaries), Ann. Inst. Fourier (Grenoble) 62 (2012), no. 3, 1045-1080, DOI 10.5802/aif.2716. MR3013816 
[29] P. Kim, R. Song, and Z. Vondraček, Two-sided Green function estimates for killed subordinate Brownian motions, Proc. Lond. Math. Soc. (3) 104 (2012), no. 5, 927-958, DOI 10.1112/plms/pdr050. MR2928332

[30] P. Kim, R. Song, and Z. Vondraček, Uniform boundary Harnack principle for rotationally symmetric Lévy processes in general open sets, Sci. China Math. 55 (2012), no. 11, 2317-2333, DOI 10.1007/s11425-012-4516-6. MR.2994122

[31] P. Kim, R. Song, and Z. Vondraček, Global uniform boundary Harnack principle with explicit decay rate and its application, Stochastic Process. Appl. 124 (2014), no. 1, 235-267, DOI 10.1016/j.spa.2013.07.007. MR3131293

[32] P. Kim, R. Song, and Z. Vondraček, Boundary Harnack principle and Martin boundary at infinity for subordinate Brownian motions, Potential Anal. 41 (2014), no. 2, 407-441, DOI 10.1007/s11118-013-9375-4. MR 3232031

[33] P. Kim, R. Song, and Z. Vondraček, Martin boundary for some symmetric Lévy processes, Festschrift Masatoshi Fukushima, Interdiscip. Math. Sci., vol. 17, World Sci. Publ., Hackensack, NJ, 2015, pp. 307-342, DOI 10.1142/9789814596534_0017. MR3379348

[34] H. Kunita and T. Watanabe, Markov processes and Martin boundaries. I, Illinois J. Math. 9 (1965), 485-526. MR0181010 (31 \#5240)

[35] J. Lelong-Ferrand, Etude au voisinage de la frontière des fonctions subharmoniques positives dans un demi-espace (French), Ann. Sci. École Norm. Sup. (3) 66 (1949), 125-159. MR.0031603 (11,176f)

[36] V. G. Maz'ja, On Beurling's theorem on the minimum principle for positive harmonic functions (Russian), Investigations on linear operators and the theory of functions, III. Zap. Naučn. Sem. Leningrad. Otdel. Mat. Inst. Steklov. (LOMI) 30 (1972), 76-90. MR0330484 (48 \#8821)

[37] I. Miyamoto and H. Yoshida, Two criterions of Wiener type for minimally thin sets and rarefied sets in a cone, J. Math. Soc. Japan 54 (2002), no. 3, 487-512, DOI 10.2969/jmsj/1191593906. MR1900954 (2003d:31002)

[38] L. Naïm, Sur le rôle de la frontière de R. S. Martin dans la théorie du potentiel (French), Ann. Inst. Fourier, Grenoble 7 (1957), 183-281. MR0100174 (20 \#6608)

[39] K.-i. Sato, Lévy processes and infinitely divisible distributions, Cambridge Studies in Advanced Mathematics, vol. 68, Cambridge University Press, Cambridge, 1999. Translated from the 1990 Japanese original; Revised by the author. MR.1739520 (2003b:60064)

[40] P. Sjögren, Une propriété des fonctions harmoniques positives, d'après Dahlberg (French), Séminaire de Théorie du Potentiel de Paris, No. 2 (Univ. Paris, Paris, 1975), Lecture Notes in Math., vol. 563, Springer, Berlin, 1976, pp. 275-282. MR0588344 (58 \#28556)

[41] P. Sjögren, Weak $L_{1}$ characterizations of Poisson integrals, Green potentials and $H^{p}$ spaces, Trans. Amer. Math. Soc. 233 (1977), 179-196. MR0463462 (57 \#3412)

[42] R. Song and Z. Vondraček, Potential theory of subordinate Brownian motion, in Potential Analysis of Stable Processes and its Extensions, P. Graczyk and A. Stos, eds., Lecture Notes in Math., vol. 1980, 2009, pp. 87-176. MR2569321(2011i:60140)

[43] E. M. Stein, Singular integrals and differentiability properties of functions, Princeton Mathematical Series, No. 30, Princeton University Press, Princeton, N.J., 1970. MR0290095 (44 \#7280)

Department of Mathematical Sciences and Research Institute of Mathematics, Seoul National University, Building 27, 1 Gwanak-Ro, Gwanak-gu, Seoul 151-747, Republic of KOREA

E-mail address: pkim@snu.ac.kr

Department of Mathematics, University of Illinois, Urbana, Illinois 61801

E-mail address: rsong@math.uiuc.edu

Department of Mathematics, University of Zagreb, Zagreb, Croatia

E-mail address: vondra@math.hr 